\title{
Volume, Side-Area, and Force Direction of Berkovich and Cubecorner Indenters, Novel Important Insights
}

\author{
Gerd Kaupp \\ University of Oldenburg, Oldenburg, Germany \\ Email: gerd.kaupp@uni-oldenburg.de
}

How to cite this paper: Kaupp, G. (2021) Volume, Side-Area, and Force Direction of Berkovich and Cubecorner Indenters, Novel Important Insights. Advances in Materials Physics and Chemistry, 11, 212-241. https://doi.org/10.4236/ampc.2021.1111019

Received: October 11, 2021

Accepted: November 22, 2021

Published: November 25, 2021

Copyright $\odot 2021$ by author(s) and Scientific Research Publishing Inc. This work is licensed under the Creative Commons Attribution International License (CC BY 4.0).

http://creativecommons.org/licenses/by/4.0/

\begin{abstract}
The iteration-free physical description of pyramidal indentations with closed mathematical equations is comprehensively described and extended for creating new insights in this important field of research and applications. All calculations are easily repeatable and should be programmed by instrument builders for even easier general use. Formulas for the volumes and side-areas of Berkovich and cubecorner as a function of depth are deduced and provided, as are the resulting forces and force directions. All of these allow for the detailed comparison of the different indenters on the mathematical reality. The pyramidal values differ remarkably from the ones of so-called "equivalent cones". The worldwide use of such pseudo-cones is in severe error. The earlier claimed and used 3 times higher displaced volume with cube corner than with Berkovich is disproved. Both displace the same amount at the same applied force. The unprecedented mathematical results are experimentally confirmed for the physical indentation hardness and for the sharp-onset phase-transitions with calculated transition energy. The comparison of both indenters provides novel basic insights. Isotropic materials exhibit the same phase transition onset force, but the transition energy is larger with the cube corner, due to higher force and flatter force direction. This qualifies the cube corner for fracture toughness studies. Pile-up is not from the claimed "friction with the indenter". Anisotropic materials with cleavage planes and channels undergo sliding along these under pressure, both to the surface and internally. Their volumes add to the depression volume. These volumes are essential for the exemplified pile-up management. Phase-transitions produce polymorph interfaces that are nucleation sites for cracks. Technical materials must be developed with onset forces higher than the highest thinkable stresses (at airliners, bridges, etc.). This requires urgent revision of ISO 14577-ASTM standards.
\end{abstract}




\section{Keywords}

Closed Mathematical Formulas, Force Direction, Indenter Volumes and Side-Areas, Iteration-less Calculations, Equal Base-Area Cones, Pile-up, Phase-Transition-Onset and -Energy

\section{Introduction}

\subsection{The Force vs Indentation Depth Relation}

It was in 2013 and 2016 when physical and mathematical breakthroughs described (nano)indentations by taking into account the energy conservation law and the penetration volume of the immersed indenter, rather than the basal indenter area. [1] and [2] undeniably proved the validity of the experimentally found $h^{3 / 2}$ vs applied normal force $\left(F_{\mathrm{N}}\right)$ relation instead of $h^{2}$ for pyramidal and conical indentations from 2004 [3]. And already 22 linear $F_{\mathrm{N}}$ vs $h^{3 / 2}$ plots with materials from practically all types of materials had been published in [4]. This rightfully opposes to the nevertheless still common belief in incorrect " $h^{2}$ ", losing all information from it (no physical hardness, no initial surface and tip rounding effects, no phase transitions, no possible gradients). Similarly, the iteration of the loading curve exponent for best-fitting of the loading curve wipes out all these information. Still worldwide believed is the using $h^{2}$ derived ISO 14577 ASTM hardness (International Standardization Organization and American Society for Testing and Materials) from conical and pyramidal indentations that is used in available tabulations, peer-reviewed scientific publications, and industries. It is unduly defined as force over projected basal contact area. But why is that so? The applied force does not press to the basal area of the indenter! It must be the result of the still retained wrong exponent for the loading curve.

\subsection{ISO and ASTM Hardness and Modulus}

According to [5], the area function of the "perfect" Berkovich is $A_{\mathrm{hc}}=24.5 h_{\mathrm{c}}^{2}$, where $h_{\mathrm{c}}$ indicates contact height. The constant had been checked with the compliance $C$ (inverse stiffness) vs $A^{1 / 2}$ plots from the "two highest" $(120 \mathrm{mN})$ indentations in aluminium of [5]. $A_{\mathrm{hc}}$ is iteratively "refined" for the not ideal indenter to give the fitted "contact area" $A_{\mathrm{hc}}$ for other materials. At first the unloading curve exponent is iterated with 3 free parameters (fitted unloading steepness). Secondly, the area is iterated with 8 free parameters $C_{1}-C_{8}$. The first guess of them is $24.5 h_{\mathrm{c}}^{2}$ and the further 8 exponents on $h_{\mathrm{c}}$ decrease via 1 to $1 / 128$ for fit with the aluminum data. For smaller depths the fit is for the corresponding fused quartz data. That leads to the fitted contact depth $h_{\mathrm{c}}=h_{\max }-\varepsilon F_{\mathrm{Nmax}} / S$. The $\varepsilon$ is a disputed factor. The necessary stiffness $S$ is defined after differentiation of the fitted unloading curve as $\mathrm{d} F_{\mathrm{N}} / \mathrm{d} h_{\max }$, and the fitted reduced elastic modulus is $E_{\mathrm{r}}=S \pi^{1 / 2} / 2 A_{\mathrm{hc}}^{1 / 2}$. This does not consider that only part of $F_{\mathrm{N} \max }$ is responsible for the depth $h$ [1]. All of these iterations from [5] are the ISO 14577-ASTM Inter- 
national standard. They are automatically executed with the commercial indenters to provide iterated ISO-hardness $H$ [N/depth $\left.{ }^{2}\right]$.

Furthermore, the equally iterated $E_{\mathrm{r}}\left[\mathrm{N} / \mathrm{depth}^{2}\right]$ is not at all the claimed "Young's modulus". The latter is, of course, a strictly unidirectional property out of the 6 by 6 matrix of Young's moduli that finally, depending on crystal symmetries, gives $9,7,6$, or in the cubic case 3 independent always very different Young's moduli. That is generally known and communicated, but here falsely disregarded.

The common use of the false exponent on $h$ followed by exhaustive iterations and false definitions is dangerous. All of that creates false materials properties and it denies phase-transitions under load with their polymorph interfaces. Unfortunately, that is still enforced by ISO 14577-ASTM for technical materials with catastrophic risks.

\subsection{The Mathematics with the Correct Exponent $3 / 2$ on the Depth $h$}

The undeniably [2] correct physical hardness from conical and pyramidal indentations is obtained as $k\left(F_{\mathrm{N}} / h^{3 / 2}\right)$, which is the penetration resistance as slope of the experimental linear $F_{\mathrm{N}}$ vs $h^{3 / 2}$ plot (also called "Kaupp-plot" since 2004 and 2016) as Equation (1). It must be corrected for any axis cut $F_{\mathrm{a}}$ to give (2). Such axis cuts can be positive or negative due to various surface effects. They are excluded from regressions. The various reasons for initial surface effects have been amply discussed in preceding publications. Sample surfaces are not always free of layers (twins, oxides, hydroxides, chemical and mechanical pre-treatment such as polishing etc). Their separate elucidation requires indentations with very small depth ranges. Axis cuts are corrected for in a straightforward way.

It is the iteration-free and undeniable mathematics that reaches correct physical data of materials and totally new ones from (nano)indentations. Examples are the physical indentation hardness [1] [2] [3] [4], the previously undetectable phase-transitions under load as kink unsteadiness on the $F_{\mathrm{N}}$ vs $h^{3 / 2}$ plot (e.g. Figure 2 [2]), and the phase-transition energies. The phase-transition energies are now normalized per depth region. Unfortunately, the presently still used concept of "work hardening" with iterated exponential functions is meaningless, dangerous, and obsolete. The formed polymorphs can be spectroscopically identified and they create dangerous interfaces after their sharp onset with the nontransformed material. Polymorph interfaces are sites for crack nucleation.

For the mathematical description of the pyramidal or conical indentations on a physical basis the formulas from [1] are below extended and completed, and their use is outlined. For their deduction it was first necessary to distinguish applied work $W_{\text {applied }}$ from indentation work $W_{\text {indent }}$ [1]. The latter is the work for the impression. The former contains additionally the work for pressure formation to its environment and all types of plasticizing. The whole indentation process subdivides $80 \%$ of the applied force for the inverted pyramid or inverted cone formation and $20 \%$ of it for pressure and all kinds of plasticizing. This al- 
ways mathematically precise 4:1 ratio in (3) has been mathematically deduced in 2013 [1]. In the case of phase-transition upon indentation the sharp kink unsteadiness occurs in the $F_{\mathrm{N}}$ vs $h^{3 / 2}$-plot, separating linear branch 1 from linear branch 2 with different slopes $k_{1}$ and $k_{2}$. These are the different penetration resistances (hardnesses), in e.g. $\mathrm{mN} / \mu \mathrm{m}^{3 / 2}$ units of the involved polymorphs. The $h_{\text {kink }}$ and the $F_{\mathrm{N} \text {-kink }}$ values for $W_{\text {1-applied }}$ are easily obtained by equation of the regression line formulas from the two branches for the triangle Equation (4). For the $W_{2 \text {-applied }}$ value one has to integrate (2) to give (5) that is multiplied with 1.25 according to (3) and it is added to $W_{1 \text {-applied }}$ for obtaining $\Sigma W_{\text {applied. }}$. The latter is subtracted from full $W_{\text {applied }}$ to obtain the transition work $W_{\text {transition }}$ up to $h_{2}$ [1] [6] ( $h_{2}$ is freely chosen; perhaps at the depth where another kink deviation starts). The transition-energy is calculated according to (7). Its normalization is now by division through the depth difference (8) (no longer through the force difference) for better comparison of different indenter tips.

An analogous procedure is applied for multiple phase-transitions from kink to kink. That purely algebraic sequence of calculations has been performed to numerous materials by the present author for endothermic and exothermic phase transitions under load. These will now be augmented with different materials and comparison of two different indenters. Again, phase-transitions cannot be obtained by iterative fittings including finite element calculations. Their onset forces and transition energies are important for daily life security, because the formation of polymorph interfaces can be sites of catastrophic initiations of crashes.

$$
\begin{gathered}
F_{\mathrm{N}}=k h^{3 / 2} \\
F_{\mathrm{N}}=k h^{3 / 2}+F_{\mathrm{a}} \\
W_{\text {indent }}=0.8 W_{\text {applied }} \\
W_{1 \text {-applied }}=0.5 h_{\text {kink }} F_{\text {N-kink }} \\
W_{\text {2-indent }}=0.4 k_{2}\left(h_{2}^{5 / 2}-h_{\text {kink }}^{5 / 2}\right)+F_{2-\mathrm{a}}\left(h_{2}-h_{\text {kink }}\right) \\
\text { full } W_{\text {applied }}=0.5 F_{\mathrm{N}-\text { max }} h_{\text {max }} \\
W_{\text {transition }}=\text { full } W_{\text {applied }}-\Sigma\left(W_{\text {applied }}\right) \\
\text { normalized } W_{\text {transition }}=W_{\text {transition }} /\left(h_{2}-h_{\text {kink }}\right)
\end{gathered}
$$

\section{Materials and Methods}

A fully calibrated Hysitron Inc. Triboscope ${ }^{\circledR}$ Nanomechanical Test Instrument with $2 \mathrm{D}$ transducer, leveling device, and direct combination with a Nanoscope II atomic force microscope (AFM) was used for the author's (nano)indentations. Loading times were $30 \mathrm{sec}$, force-controlled in contact mode. The radii of the cubecorner $(55 \mathrm{~nm})$ and Berkovich $(110 \mathrm{~nm})$ diamond indenters [4] were directly measured by non-contact AFM at a Park NX20 atomic force microscope from Park Systems. Highly resolved microscopic images were obtained with a 
digital 3D microscope from Keyence Ltd., model VHX - $100 \mathrm{~K}$ with almost uniform coaxial vertical illumination through optical fiber, ring lenses, and $45^{\circ}$ half mirror. A CCD-camera recorded the light of the halogen lamp, as coaxially reflected back by the sample through the half mirror. The chosen focal depth steps were down to $1 \mu \mathrm{m}$.

Most preferable for the analysis of AFM loading curves is the direct calculation of all instrumental data points (about 20,000) according to (2) as loaded to Excel $^{\circledR}$. For published loading curves, with reasonable experimental description, the data digitization is obtained after paper filling enlargement with the plot digitizer 2.5.1 program (https://www.softpedia.com/) giving 50 - 70 data-pairs, or manually 20 to 30 data pairs. Visual inspection of the printed Excel ${ }^{\circledR} F_{\mathrm{N}}$ vs $h^{3 / 2}$ plots looks for the linear ranges, surface effects, obvious gradients, and other peculiarities (e.g. non-linear force application). The kink is sharp, but there might be some short soft transitions in buffering biological materials [7] or at too rapid penetration. The bearing analysis routine [8] [9] is used for the measurement of depression and pile-up volumes with respect to the plane through the respective edges and corners. We did not use the complicate formulas with numerous assumptions of [10].

Figure 1 looks quite smooth but Figure 2 shows a much extended initial effect, some minimal displaced points in the kink region and a deviation above 90 $\mathrm{mN}$ load. The initial effect study would require separate indentation to about 20 $\mathrm{mN}$ load. It is possible that there is another transition, as perhaps twinning. Endothermic twin formation of tungsten is indeed initiated by application of shear-force to tungsten [11]. The final deviation must here be excluded from further consideration, because it also occurs comparably with 6 materials all above $90 \mathrm{mN}$ load in [5]. But for $k_{1}$ and $k_{2}$ there exists no ambiguity due to correlation

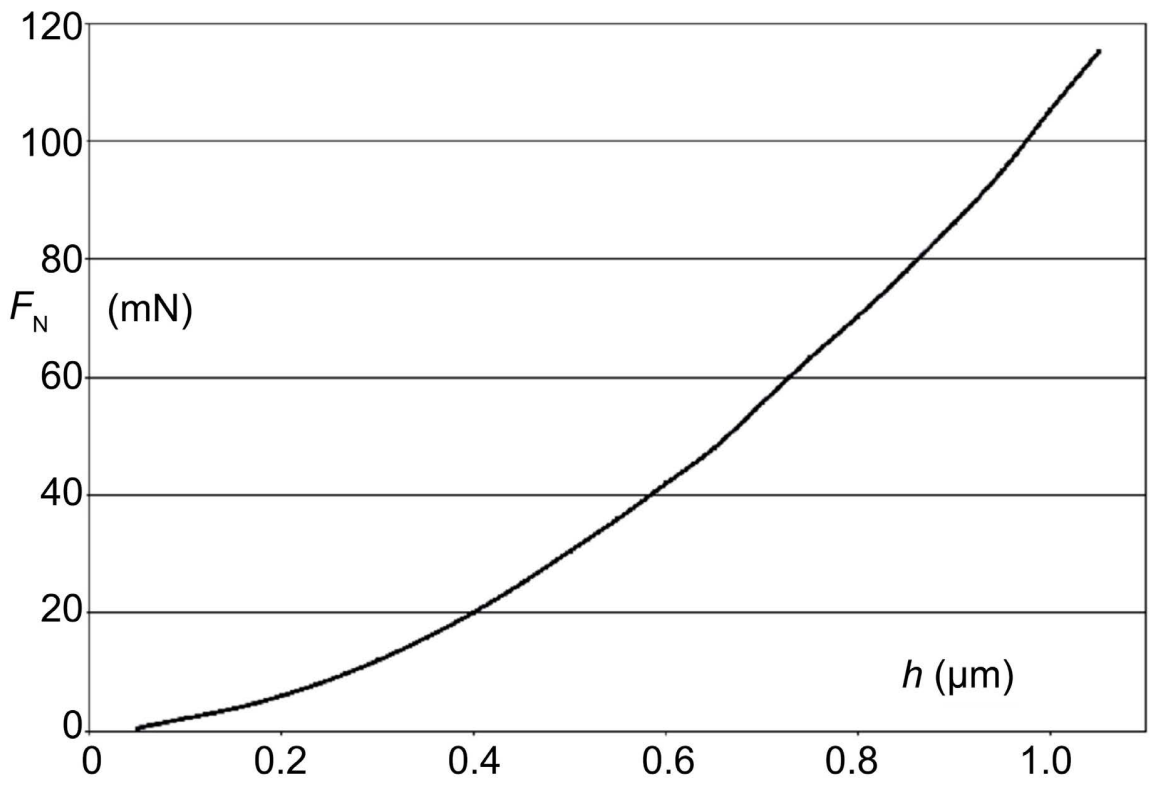

Figure 1. Force vs depth image with Berkovich onto tungsten from Figure 9 in [5]. 


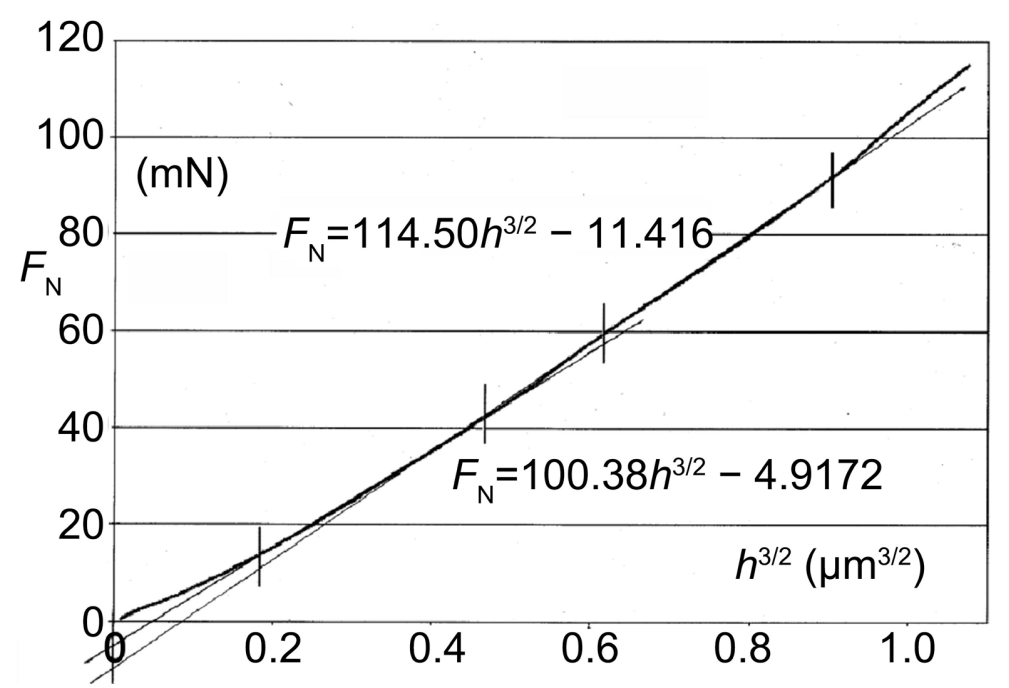

Figure 2. $F_{\mathrm{N}}$ vs $h^{3 / 2}$ plot according to (2) of the curve in Figure (1) with regression equations in the selected ranges (kink at $0.460254957 \mu \mathrm{m}^{3 / 2}$ and $41.28319258 \mathrm{mN}$ ); the slopes are the physical hardness $k\left(\mathrm{mN} / \mu \mathrm{m}^{3 / 2}\right)$; the initial part is cut off and should be separately studied with lower force range; a short intermediate range around the kink point is not part of the regression.

coefficients $\mathrm{R}^{2}$ of 0.9995 and 0.9996 . It appears to be a poor instrumental compliance above $90 \mathrm{mN}$ or non-linear force application from that point. This has been revealed in [12]. Our analysis is versatile enough to reliably determine the phase-transition energy despite such particular difficulties. This technique is not of the Arrhenius type and does not require indentations at different temperatures as with activation energies from indentations as in [13].

The formulas (1) through (7) contain all information, but a step to step description of the calculation is also necessary. Due to exponents, sums and differences it appears necessary to calculate with 10 figures, so that the experimental errors are not increased by the calculations. For the transition energies we equate the regression lines ( $\mathrm{R}^{2}$ must be $\left.>0.9995\right) \quad F_{\mathrm{N}-1}=k_{1} h^{3 / 2}+F_{\mathrm{a}-1}$ and $F_{\mathrm{N}-2}=k_{2} h^{3 / 2}+F_{\mathrm{a}-2}$ to obtain $h_{\text {kink }}^{3 / 2}, h_{k \text { kinh }} h_{\text {kink }}^{5 / 2}$, and $F_{\mathrm{N}-\text { kink. }}$. $W_{1 \text {-applied }}$ is then calculated according to (4). $W$ stands for work or energy. The subscript "kink" describes the sharp intersection of the regression lines that is the "kink-unsteadiness" at the onset point (depth and force) of the phase-transition.

The force value $F_{\text {end }}\left(>F_{\mathrm{N}-1}\right)$ for the calculation of $W_{2 \text {-applied }}$ can be chosen at will (in the case of a following higher phase-transitions one chooses the value for $h_{\max }$ $\left.=h_{\text {kink-2 }}\right)$. One then calculates $h_{\max }^{3 / 2}, h_{\max }$ and $h_{\max }^{5 / 2}$ for $F_{\mathrm{N}-\max }=k_{2} h_{\max }^{3 / 2}+F_{\mathrm{a}-2}$. With these and obtains $\Delta h^{5 / 2}=h_{\max }^{5 / 2}-h_{\text {kink }}^{5 / 2}$ and $\Delta h=h_{\max }-h_{\text {kink }}$. One has thus all mathematical values for the calculation of the indentation work. $W_{2 \text {-indent }}$ according to (5) gives $W_{\text {-applied }}=1.25 W_{\text {2-indent }}$ according to (3), so that $\Sigma W_{\text {applied }}$ can be formed. The value of full $W_{\text {applied }}$ is $0.5 F_{\text {Nend }} h_{\text {end }}$, because the previous calculations corrected for both axes cut $F_{\mathrm{a}-1}$ and $F_{\mathrm{a}-2}$.

The transition-energy (7) $W_{\text {transition }}=$ full $W_{\text {applied }}-\Sigma W_{\text {applied }}$ is then divided by $\Delta h$ to finally obtain normalized $W_{\text {transition }} / \mu \mathrm{m}(\mathrm{mN} \mu \mathrm{m} / \mu \mathrm{m})$ values. These charac- 
terize the phase-transition onset of the material in question. Different materials can be compared and calculations for any wished depths and forces are possible. A corresponding sequence applies for higher phase-transitions at higher forces.

\section{Results}

\subsection{Verification of the Transition-Energy Calculations}

The determinations of the phase-transition energies are tested at a practical example from the literature. For example, Figure 1 shows the $F_{\mathrm{N}}$ vs $h$ curve that is the basis of the still used iterative ISO-hardness $\left(H_{\text {ISO }}\right)$ and ISO-modulus of tungsten, which is used as indentation standard for calibrations up to $120 \mathrm{mN}$, even though compliance or linearity are unfortunately miscalibrated (also for the other standard materials in [5]) and despite the particularly pronounced flaws that are challenged in the Introduction. We open the possibility for nevertheless using such published experimental loading curves for the non iterative penetration resistance $\left(k \mathrm{mN} / \mu \mathrm{m}^{3 / 2}\right)$ calculation and detection of phase-transitions with their onset force and phase-transition energy in the valid regions.

The purely arithmetic calculations remove the initial effects, less precise data-pairs close to the kink point, and deviations due to non-linear force applications. After these strict precautions it can be tested whether the same result is obtained for $W_{\text {transition }} / \mu \mathrm{m}$ when the different selected forces at 60 and $90 \mathrm{mN}$ are chosen from the tungsten curves in the Figure 1 and Figure 2. Table 1 collects the test results as calculated according to (1)-(8) with 10 figures. The calculated values of $W_{\text {transition }} / \Delta h$ from the different end forces confirm the correctness of our formula scheme. The very low differences of $0.665 \times 10^{-6} \%$ (due to rounding) also confirm that the errors by the calculation using exponents, summations and subtractions strongly disappear in relation to unavoidable experimental errors.

Table 1. Calculation test from $F_{\mathrm{N}-1}=100.38 h^{3 / 2}-4.9172$ and $F_{\mathrm{N}-2}=114.50 h^{3 / 2}-11.416$ for $W_{\text {transition }}$ of tungsten at different chosen end forces from Figure 2.

\begin{tabular}{ccc}
\hline$F_{\text {end }}$ & $60(\mathrm{mN})$ & $90(\mathrm{mN})$ \\
\hline$h_{\text {kink }}(\mu \mathrm{m})$ & 0.596118111 & 0.596118111 \\
$F_{\text {Nkink }}(\mathrm{mN})$ & 41.28319258 & 41.28319258 \\
$W_{\text {applied-1 }}(\mathrm{mN} \mu \mathrm{m})$ & 12.30482939 & 12.30482939 \\
$\Delta h(\mu \mathrm{m})$ & 0.133888326 & 0.326171522 \\
$\Delta h^{5 / 2}\left(\mu \mathrm{m}^{5 / 2}\right)$ & 0.180953682 & 0.542532597 \\
$W_{\text {indent-2 }}(\mathrm{mN})$ & 6.759209506 & 21.12441884 \\
$W_{\text {applied-2 }}(\mathrm{mN} \mu \mathrm{m})$ & 8.449011820 & 26.40552356 \\
$\Sigma W_{\text {applied }}(\mathrm{mN} \mu \mathrm{m})$ & 20.75384127 & 38.71035295 \\
Full $W_{\text {applied }}(\mathrm{mN} \mu \mathrm{m})$ & 21.90019311 & 41.50303349 \\
$W_{\text {transition }}(\mathrm{mN} \mu \mathrm{m})$ & 1.14635184 & 2.792680535 \\
$W_{\text {transition }} / \Delta h(\mathrm{mN} \mu \mathrm{m} / \mu \mathrm{m})$ & 8.561999946 & 8.561999889
\end{tabular}


It is therefore advisable to perform a computer program for the calculation of phase-transition energies in the future. However, the independent expert scrutiny by using the physically correct $F_{\mathrm{N}}$ vs $h^{3 / 2}$ plot of the experimental data from conical or pyramidal indentations remains indispensable. The situation with unfitted data of spherical indentations is described in [14] and [15].

This Table 1 indicates the calculation procedure and the precision of the calculations with 10 significant figures for at the end obtaining reasonably rounded results. The normalization of the transition energy at the onset kink point is now per depth region $\left(h_{\text {final }}-h_{\text {kink }}\right)$.

\subsection{Phase-Transition Energies}

It is important to distinguish the modification of the indented material and the indented surface of crystals. For example quartz $\left(\mathrm{SiO}_{2}\right)$ occurs as amorphous fused quartz and $\alpha$-quartz (rocksalt), the latter with surface twins that can be cautiously removed by polishing if necessary. Also water layers will form at ambient atmosphere. Furthermore, Cristobalite, Coesite, Stishovite, and Seifertite are at ambient conditions metastable polymorphs of quartz. The latter two have been synthesized and are also known as Meteor crystals. The indentation onto Stishovite produced probably Seifertite and another still higher energetic as yet unknown polymorph, but none of the lower energy polymorphs of $\mathrm{SiO}_{2}$. All of these give different results upon indentation [6].

The amorphous to amorphous transition of fused quartz is well known. Nevertheless the present ISO 14577 still uses fused quartz for indentation-instrument calibrations, which is another source of error when not taking care of it. The phase-transition energies can be negative (exothermic) or mostly positive (endothermic). The exothermic ones of iron (100) and (110) as well as of InGaAs have been published in [12] and [16]. Also the 5 indented different faces of $\alpha-\mathrm{SiO}_{2}$ undergo the phase-transition exothermically [12] and [16].

The already published indentations with endothermic phase-transitions of superalloys including aluminium and $\gamma \mathrm{Ti}-6 \mathrm{Al}-4 \mathrm{~V}$ in [16] are of particular importance in view of flying safety and require most scrutiny. Light titanium-aluminium-vanadium alloys are the preferred materials for airplane constructions. The present author complained in his publication [16] and in preceding worldwide lectures the low phase-transition onset forces and the low phase-transition energy of $\gamma \mathrm{Ti}-6 \mathrm{Al}-4 \mathrm{~V}$ alloy that are much inferior with respect to pure aluminum and superalloys. For example, the phase-transition onset of the Vitreloy105 metallic glass occurs at 58 fold higher onset force and at 108-fold higher phase-transition energy. All of the various researchers who indented onto and published on this and related TiAlX alloys did not check for phase transition onsets, but they persisted on the unphysical ISO-ASTM standards. They are urgently asked to reanalyze and publish their original experimental data. The present author urgently exacted and exacts that the builders of airliners must check and improve their technical alloys by physical indentation $\left(h^{3 / 2}\right.$ not $h^{2}$ and no itera- 
tions). Phase-transitions under load must be recognized, detected, and characterized. Their onset force and transition energy must be increased with improved alloys for much higher phase-transition onset forces and transition energies. Phase-transition polymorph interfaces are crack nucleation sites that must be avoided upon operation [17]. The justification of these urgent reports followed soon: propeller blades, one with a deadly accident, broke twice from a turbine and hit the fuselage within one year from the same type of airliner. Obviously alerted by [16] and [17] hundreds of airliners were together grounded for months due to scratches even at the pickle forks between wings and fuselage, but several catastrophic airliner accidents still occurred. Unfortunately, sharp onset phase-transitions under mechanical load with their polymorph interfaces formations are not detectable with the disproved [2], but still binding ISO-ASTM standards that continue to be an enforcing part of the industrial certification. This must be urgently and immediately changed, for safer flying and safer daily life. ISO and ASTM representatives are being personally informed since long ago.

Numerous further phase-transition onsets and endothermic transition energies are published in [16] for calibration standards, silicon (two faces), strontium titanate (3 faces), numerous salts, polymers, wood, and organic crystals. These cannot be repeated here.

\subsection{The Comparison of Berkovich with Cubecorner Phase Transitions Using the Indenter Volumes and Side-Areas}

While the Berkovich indenter is ISO standard in indentation testing, the steeper cubecorner has advantages for the study of fracture toughness. Actually, the cubecorner appears more appropriate for the fracture toughness measurement by indentation and we can explain it. Fracture toughness is commonly calculated as $K=0.036(E / H)^{1 / 2}\left(F_{\mathrm{N}} / c^{3 / 2}\right)$ where $c$ is the crack length, $H$ and $E$ are ISO hardness and ISO falsely so-called "Young's elastic modulus". The 0.036 is an empirical constant "from a fit" for cubecorner, but the $E / H$ values are taken from Berkovich indentations [18]. Clearly, Berkovich and cubecorner indentations provide different results. The strange claims in that paper of the Oliver-Pharr group that the "hardness measured with the two indenters should be about the same" and the unpardonable claim that "the cubecorner geometrically displaces more than 3 times the volume of the Berkovich" at the same force are nevertheless unduly acknowledged. For example, it is used in [19] with citation of [18]. Also Wang [20] falsely claims that the cube-corner transforms more than 3 times the volume of the Berkovich "for a given load, and thus produces "higher stress beneath the indenter". On the other hand [21] claims that "the Berkovich indenter probes a volume approximately 8-times bigger than the cube-corner" and "the Berkovich indenter distributes the load over a wider area with respect to the sharp cube-corner indenter". All of these contradictionary published claims are more than disturbing. Clearly, the physical hardness $(k-$ 
value as slope of the $F_{\mathrm{N}}$ vs $h^{3 / 2}$ plot [2]) and indentation modulus from cubecorner and Berkovich results are different. Also ISO-hardness $H$ and ISO-socalled "Young's" modulus $E$ depend on the indenter acuteness [21]. Numerous fitting simulations exist, but experimental comparisons on the basis of published experimental loading curves are rare. It was however not taken into account, that the cubecorner must create the same indentation volume (plus eventual pile-up volume) than the Berkovich at the same force, because the energy law cannot be disregarded. The same applied work creates the same volume. This can be seen in Table 2 and Table 3. It is not seen by Bor et al. [21] who simulated fracture toughness again with $H$ and $E$ from Berkovich and cracking with cubecorner on the energy law violating 1:3 volume ratio basis with Berkovich and cubecorner. This paper tries to explain the differences upon indentations with the two-dimensional shape of broader Berkovich and more acute cubecorner and a poorly comprehensible "densification" of material at the steeper cubecorner apex. Clearly, the obvious fact is not considered that the material is displaced at the faces of the tip that penetrates much deeper than the Berkovich. Inverse triangular pyramids are formed in the material with different bases and depths. And again, their totally displaced volume must be equal at the same applied force, as the energy law must not be violated (see also Section 3.5). Thus, we cannot agree at all with the lengthy argumentation in [21].

It appears urgently important to deduce an undeniable mathematical basis for the explanation of the remarkable differences between the indenters, despite of the same displaced volume (including eventual pile-up volume). There were no mathematical formulas for the volumes of Berkovich and cubecorner diamond indenters as a function of depth. These pyramids are characterized with "equivalent" cone angles and with the known angles $\beta$ from centerline to face (e.g. [21]). Unfortunately, the reason for different results has never been discussed with respect to indenter volumes. These angles $\beta$ are $65.27^{\circ}$ for Berkovich and $35.264^{\circ}$ for cubecorner [22]. They have obviously never been used for the calculation of

Table 2. Influence of indenter volume and side-areas of the indenter pyramids according to Equations (13) and (14), as tested with the force-depth curves of Zerodur ${ }^{\circledR}$ in figure 10 of [23].

\begin{tabular}{ccccccc}
\hline $\begin{array}{c}\text { Force } F_{\mathrm{N}} \\
(\mathrm{mN})\end{array}$ & $\begin{array}{c}\text { Berkovich } \\
h(\mu \mathrm{m})^{\mathrm{a})}\end{array}$ & $\begin{array}{c}\text { Cubecorner } \\
h(\mu \mathrm{m})\end{array}$ & $\begin{array}{c}\text { Berkovich } \\
V\left(\mu \mathrm{m}^{3}\right)\end{array}$ & $\begin{array}{c}\text { Cubecorner } \\
V\left(\mu \mathrm{m}^{3}\right)\end{array}$ & $\begin{array}{c}\text { Berkovich } \\
3 A\left(\mu \mathrm{m}^{2}\right)\end{array}$ & $\begin{array}{c}\text { Cubecorner } \\
3 A\left(\mu \mathrm{m}^{2}\right)\end{array}$ \\
\hline 100 & 0.959 & 2.0300 & 7.2011 & 7.2446 & 24.8016 & 18.5443 \\
200 & 1.380 & 2.9167 & 21.4580 & 21.4882 & 51.3570 & 39.0741 \\
300 & 1.718 & 3.6333 & 41.4048 & 41.5357 & 79.5954 & 59.4046 \\
400 & 1.974 & 4.1806 & 62.8046 & 63.2753 & 105.0838 & 78.6493 \\
500 & 2.221 & 4.6944 & 89.4531 & 89.5897 & 133.0267 & 99.1694 \\
600 & 2.449 & 5.1667 & 119.9268 & 119.4075 & 161.7407 & 120.1279 \\
\hline
\end{tabular}

a) The final depths at $600 \mathrm{mN}$ were $2.42769 \mu \mathrm{m}$ for Berkovich and $5.15639 \mu \mathrm{m}$ for cubecorner. 
Table 3. Comparison of Berkovich and cubecorner indentations from published loading curves; the calculations are with the units of the $k$-values followed by transformation into $\mathrm{mN}$ and $\mu \mathrm{m}$ units and rounding from initially 10 significant figures.

\begin{tabular}{|c|c|c|c|c|c|c|}
\hline $\mathrm{n}$ & Material & $\begin{array}{l}k_{1} \\
k_{2}\end{array}$ & $h_{\text {trans onset }}(\mu \mathrm{m})$ & $F_{\text {trans onset }}(\mu \mathrm{m})$ & $\begin{array}{c}W_{\text {transition }} \\
(\mathrm{mN} \mu \mathrm{m} / \Delta h)\end{array}$ & Data Source \\
\hline 1 & $\begin{array}{l}\text { Zerodur } \\
\text { Berkovich }\end{array}$ & $\begin{array}{l}141.81 \mathrm{mN} / \mu \mathrm{m}^{3 / 2} \\
189.24\end{array}$ & 1.54678 & 248.2246 & 86.8650 & Ceram Internat, Elsevier, \\
\hline 2 & $\begin{array}{l}\text { Zerodur } \\
\text { Cubecorner }\end{array}$ & $\begin{array}{l}46.121 \mathrm{mN} / \mu \mathrm{m}^{3 / 2} \\
62.230\end{array}$ & 3.33355 & 250.1074 & 96.4875 & $2016,42,12740$, Figure 10 \\
\hline 3 & $\begin{array}{l}\text { Fused } \mathrm{SiO}_{2} \\
\text { Berkovich }\end{array}$ & $\begin{array}{l}1.5289 \mathrm{uN} / \mathrm{nm}^{3 / 2} \\
1.8347\end{array}$ & 0.09016 & 1.19808 & 0.22610 & Hysitron Handbook \\
\hline 4 & $\begin{array}{l}\text { Fused } \mathrm{SiO}_{2} \\
\text { Cubecorner }\end{array}$ & $\begin{array}{l}0.4480 \mu \mathrm{N} / \mathrm{nm}^{3 / 2} \\
0.5561\end{array}$ & 0.18883 & 1.12375 & 0.23926 & $\begin{array}{l}\text { Int J Mater Res } 200596 \text {, } \\
1226\end{array}$ \\
\hline 5 & $\begin{array}{l}\mathrm{Na}_{2} \mathrm{O}-\mathrm{Al}_{2} \mathrm{O}_{3}-\mathrm{SiO}_{2} \\
\text { Glass Berkovich }\end{array}$ & $\begin{array}{l}1.8868 \mu \mathrm{N} / \mathrm{nm}^{3 / 2} \\
2.2632\end{array}$ & 0.13679 & 3.10289 & 0.388391 & J Amer Ceram Soc \\
\hline 6 & $\begin{array}{l}\mathrm{Na}_{2} \mathrm{O}-\mathrm{Al}_{2} \mathrm{O}_{3}-\mathrm{SiO}_{2} \\
\text { Glass Cubecorner }\end{array}$ & $\begin{array}{l}0.4765 \mu \mathrm{N} / \mathrm{nm}^{3 / 2} \\
0.6119\end{array}$ & 0.36145 & 3.08279 & 0.84159 & 2018 101, 2930, Figure 2(A) \\
\hline 7 & $\begin{array}{l}\text { 2C22 Steel stress-free, } \\
\text { Berkovich }\end{array}$ & $\begin{array}{l}45.814 \mathrm{mN} / \mu \mathrm{m}^{3 / 2} \\
65.225\end{array}$ & 0.92967 & 34.20895 & 14.4435 & Procedia Engineering \\
\hline 8 & $\begin{array}{l}\text { 2C22 Steel stress-free, } \\
\text { Cubecorner }\end{array}$ & $\begin{array}{l}9.1998 \mathrm{mN} / \mu \mathrm{m}^{3 / 2} \\
13.139\end{array}$ & 2.38606 & 33.29274 & 11.35050 & $2011,10,3528$, Figure 1(b) \\
\hline 9 & $\begin{array}{l}\text { Nickel } \\
\text { Berkovich }\end{array}$ & $\begin{array}{l}127.56 \mathrm{mN} / \mu \mathrm{m}^{3 / 2} \\
167.96\end{array}$ & 0.60051 & 52.75485 & 19.05449 & Philosophical Magazine \\
\hline 10 & $\begin{array}{l}\text { Nickel } \\
\text { Cubecorner }\end{array}$ & $\begin{array}{l}25.124 \mathrm{mN} / \mu \mathrm{m}^{3 / 2} \\
31.417\end{array}$ & 1.05640 & 26.24538 & 5.899987 & $2016,96,3442$ \\
\hline 11 & $\begin{array}{l}\text { Germani }{ }^{2} \\
\text { Berkovich }\end{array}$ & $\begin{array}{l}97.083 \mathrm{mN} / \mu \mathrm{m}^{3 / 2} \\
128.58\end{array}$ & 0.27330 & 12.53433 & 4.377296 & Appl Phys Lett 2005, 86, \\
\hline 12 & $\begin{array}{l}\text { Germanium } \\
\text { Cubecorner }^{c)}\end{array}$ & $29.085 \mathrm{mN} / \mu \mathrm{m}^{3 / 2}$ & & & & 131907 \\
\hline 13 & $\begin{array}{l}\mathrm{Cu}_{60} \mathrm{Zr}_{30} \mathrm{Ti}_{10}{ }^{\mathrm{d})} \\
\text { Berkovich }\end{array}$ & $\begin{array}{l}2.1803 \mu \mathrm{N} / \mathrm{nm}^{3 / 2} \\
2.6791\end{array}$ & 0.15718 & 4.01683 & 0.969275 & $\begin{array}{l}\text { Mater Sci Engin A 2006, } \\
430,350\end{array}$ \\
\hline 14 & $\begin{array}{l}\text { Vitreloy-105e) } \\
\text { Cubecorner }\end{array}$ & $\begin{array}{l}29.652 \mathrm{mN} / \mu \mathrm{m}^{3 / 2} \\
36.139\end{array}$ & 2.53297 & 107.9909 & 28.29528 & $\begin{array}{l}\text { Phil Mag 2006, 86, } \\
5715-5728\end{array}$ \\
\hline
\end{tabular}

a) Now calculated with all of the 475 data points; ${ }^{\text {b) }}$ sodium aluminosilicate glass; ${ }^{\mathrm{c}}$ the published cubecorner loading curve cannot be analyzed for $k_{2}$ due to rough not repairable pop-in; ${ }^{\text {d) }}$ a second transition is at about $9 \mathrm{mN}$ and $0.25 \mu \mathrm{m}$; ${ }^{\text {e) }} \mathrm{Zr}_{41} \mathrm{Ti}_{14} \mathrm{Cu}_{12.5} \mathrm{Ni}_{10} \mathrm{Be}_{22.5}$.

the indenter volumes as a function of the measured depths $h$. We need them to relate the normalized $W_{\text {transition }}$ values from indentations with the different indenters and for understanding the differing mechanical parameters. These include physical hardness, transition onset and transition-energy. Unfortunately, published experimental comparative loading curves with good precision for both indenters and suitable indentation forces at related or better equal force ranges are not very abundant. But the values from Table 3 indicate viable examples for the mathematical evaluation. The inclined 3D-sketch of an inverted pyramid in Figure 3 with the equal-sided basal triangle on top indicates how the volume 


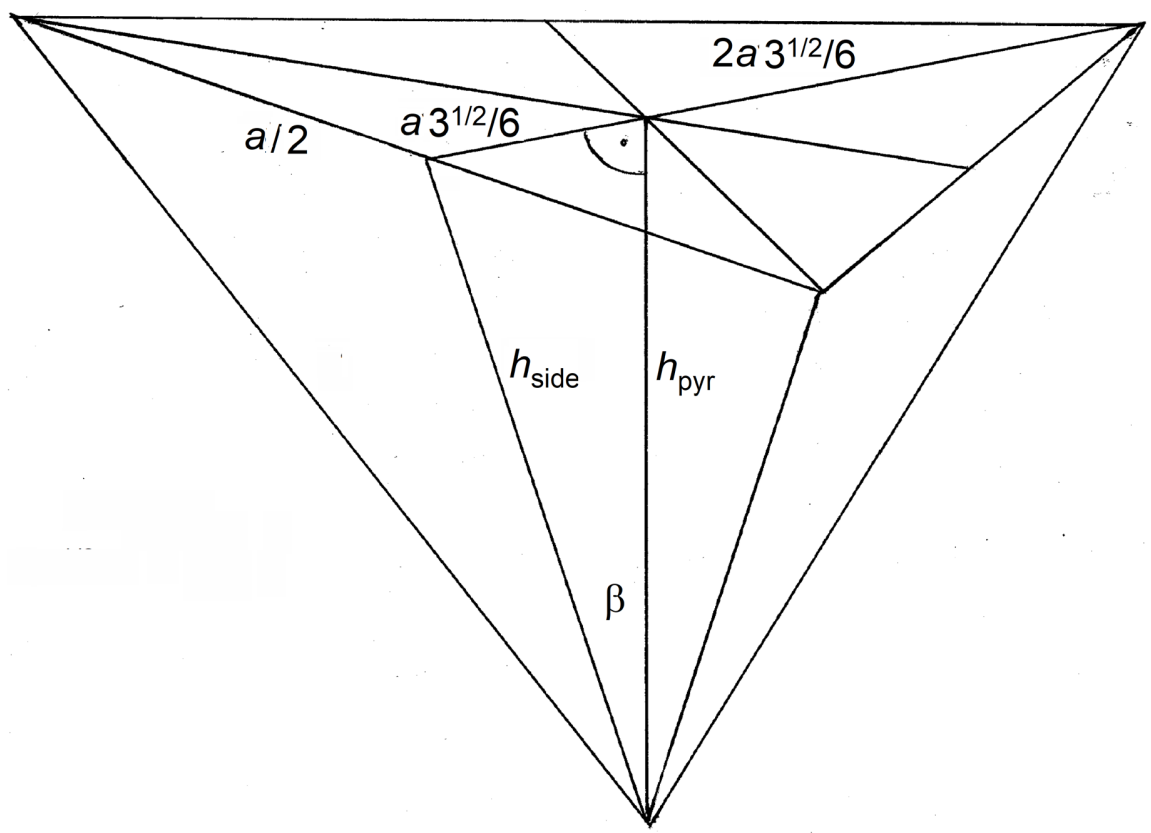

Figure 3. Perspective view on a 3D wire model of an inverted straight three-sided pyramid without crossing lines. The triangles of the model are of course not distorted. The curve with a point shall indicate that there is actually a right angle at that site.

and side-area of three-sided straight pyramids as a function of the angle $\beta$ can be mathematically described on the basis of elementary geometric formulas, by using basic trigonometry.

For the volumes of three-sided Berkovich and cubecorner we use the mathematical formula for the area of the basal equal sided triangle $\left(A_{\text {triangle }}=a^{2} 3^{1 / 2} / 4\right)$ and for the volume of the pyramid ( $\left.V_{p y r}=A_{\text {triangle }} h_{\text {pyr }} / 3\right)$, The central side length a-value has to be translated into the $h_{\mathrm{pyr}}$-value of the pyramid with the aid of the characteristic $\beta$-angle values of the pyramids $\left(\beta=65.27^{\circ}\right.$ for Berkovich and $35.264^{\circ}$ for cubecorner).

For obtaining the requested formula we have to consider that the equal sided basal triangle height $\left(h_{\text {triangle }}=a \cdot 3^{1 / 2} / 2\right)$ subdivides in the $1: 2$ ratio at the center of the triangle, so that the short part $1 / 3 h_{\text {triangle }}=a \cdot 3^{1 / 2} / 6$ is used for $\tan \beta=a \cdot 3^{1 / 2} / 6 h_{\mathrm{pyr}}$. One isolates $a$ and obtains $a^{2}=36 \tan ^{2} \beta h_{\mathrm{pyr}}^{2} / 3$. By substitution of $a^{2}$ into $A_{\text {triangle }}$ one obtains $A_{\text {triangle }}=3 \cdot 3^{1 / 2} \tan ^{2} \beta h_{\mathrm{pyr}}^{2}$ and with $V=A_{\text {triangle }} h_{\text {pyr }} / 3$ one obtains $V_{\text {pyr }}=3^{1 / 2} \tan ^{2} \beta h_{\mathrm{pyr}}^{3}$ (9). The volumes of Berkovich $\left(\beta=65.27^{\circ}\right)$ calculate thus as (10) for Berkovich and (11) for cubecorner ( $\beta$ $\left.=35.264^{\circ}\right)$. Figure 3 facilitates the survey of the trigonometric steps.

$$
\begin{gathered}
V_{\text {pyramid }}=3^{1 / 2} \tan ^{2} \beta h_{\mathrm{pyr}}^{3} \\
V_{\text {Berkovich }}=8.1647816 h_{\mathrm{pyr}}^{3} \\
V_{\text {cubecorner }}=0.86600004 h_{\mathrm{pyr}}^{3}
\end{gathered}
$$

Another point is the relation of the side-areas as a function of indentation depth that must be considered. The complete side-area $3 A_{\text {side }}$ of the pyramids has 
not been used previously. We also determine their mathematical formula for checking how these depend on the tip angle. We use the mathematical formula for the areas of isosceles triangular side faces of the pyramid area that is $A_{\text {side }}=a h_{\text {side }} / 2$. As above we first determine $a=6 \tan \beta h_{\text {pyr }} / 3^{1 / 2}$. For obtaining $h_{\text {side }}$ we use $\sin \beta=a \cdot 3^{1 / 2} / 6 h_{\text {side }}$, isolate $h_{\text {side }}$ and substitute a twice to obtain after shortening out $h_{\text {side }}=\tan \beta h_{\text {pyr }} / \sin \beta$ these factors for $a$ and $h_{\text {side }}$ are in the formula for $A_{\text {side. }}$ This gives $3 A_{\text {side }}$ for the Equations (12), or (13) and (14) with the numerical factors, for the complete inserted pyramidal side-faces.

$$
\begin{gathered}
3 A_{\text {side }}=3 \cdot 3^{1 / 2} \tan ^{2} \beta h_{\text {pyr }}^{2} / \sin \beta \\
3 A_{\text {Berkovich }}=26.96756 h_{\text {Berkovich }}^{2} \\
3 A_{\text {cubecorner }}=4.50005 h_{\text {cubecorner }}^{2}
\end{gathered}
$$

We check now the experimental loading data of isotropic Zerodur ${ }^{\circledR}$ with great detail (calculation of the irrational numbers with 10 digits before reasonable rounding) in order to tell whether the inevitable tip rounding at the apex of sharp commercial quality indenters (radii about $50 \mathrm{~nm}$ for cubecorner and 100 $\mathrm{nm}$ for Berkovich) can be neglected, due to the always executed necessary axis-cut corrections. They can be neglected with this excellent published data. Clearly, blunt or broken indenters will not provide useful data; and simulated loading curves can be excluded when the calculated displaced volumes are not equal between the different pyramids or cones at the same force (but see Section 3.5).

The regression data as calculated from the load-depth curves in figure 10 of [23] for the calculation of the ceramic Zerodur properties are

$$
F_{\mathrm{N}-1-\text { Berkovich }}=141.81 h^{3 / 2}-24.578, \quad F_{\mathrm{N}-2 \text {-Berkovich }}=189.24 h^{3 / 2}-115.82 \text { and }
$$

$F_{\mathrm{N}-1 \text {-cubecorner }}=46.121 h^{3 / 2}-30.604, F_{\mathrm{N}-2 \text {-cubecorner }}=62.23 h^{3 / 2}-128.65$. The index 1 is before phase-transition, the index 2 after phase transition. The phase-transition onset is at $248.2246 \mathrm{mN}$ for Berkovich and $250.1074 \mathrm{mN}$ for cubecorner, which is practically the same value. The normalized transition energies are for Berkovich $86.8650 \mathrm{mN} \mu \mathrm{m} / \Delta h$, and for cubecorner they are $96.4875 \mathrm{mN} \mu \mathrm{m} / \Delta h$.

The corrections for the axis cuts $F_{\mathrm{a}-1}$ and $F_{\mathrm{a}-2}(2)$, which also include tip rounding, influence the calculation results considerably. They must therefore also be reported with the complete regression line equations.

The indentation onto fused quartz $(\mathrm{n} 3-\mathrm{n} 4)$ with Berkovich $(\mu \mathrm{N}, \mathrm{nm})$ gives the regression lines $F_{\mathrm{N} 1}=1.5289 h^{3 / 2}-110.88$ and $F_{\mathrm{N} 2}=1.8347 h^{3 / 2}-372.69$. The transition onset is at $1198.0844 \mu \mathrm{N}$ load. The cubecorner $(\mu \mathrm{N}, \mathrm{nm})$ [24] gives by calculation with all of the 475 data points from the loading curve the regression lines $F_{\mathrm{N} 1}=0.4480 h^{3 / 2}-38.762$ and $F_{\mathrm{N} 2}=0.5561 h^{3 / 2}-319.010$. The transition onset is at $1123.7482 \mu \mathrm{N}$ load.

For sodium aluminosilicate glass (n5 - n6) exist indentations with Berkovich and cubecorner in the same paper [19]. The published loading curve (Raw GlassAir side) $(\mu \mathrm{N}, \mathrm{nm})$, as indented with Berkovich give the regression line $F_{\mathrm{N}-1}=1.88868 h^{3 / 2}+84.351$ and $F_{\mathrm{N}-2}=2.2632 h^{3 / 2}-517.82$ with a transition on- 
set at $3102.8851 \mu \mathrm{N}$ load. The loading curve (Raw glass-Air side) with cubecorner (only usable up to 5000 of $9000 \mu \mathrm{N}$ ) gives the following regression lines: $F_{\mathrm{N} 1}=0.4551 h^{3 / 2}-124.09$ and $F_{\mathrm{N} 2}=0.581 h^{3 / 2}-879.33$ and the transition onset is at $2605.9316 \mu \mathrm{N}$ load.

The Indentation of stress-free steel 2C22 (n7 - n8) has also been studied with both indenters $(\mathrm{mN}, \mu \mathrm{m})$ [25]. The published loading curve for Berkovich gives the regression lines $F_{\mathrm{N}-1}=45.814 h^{3 / 2}-1.8582$ and $F_{\mathrm{N}-2}=65.225 h^{3 / 2}-19.258$ $\mathrm{mN}$, and the one with cubecorner $F_{\mathrm{N}-1}=9.1998 h^{3 / 2}+0.6152$ and $F_{\mathrm{N}-2}=13.139 h^{3 / 2}-15.134 \mathrm{mN}$.

The indentation of nickel (n9 - n10) from [26] gives the regression lines from the published loading curve with Berkovich $F_{\mathrm{N}-1}=127.56 h^{3 / 2}-6.6057$ and $F_{\mathrm{N}-2}=167.96 h^{3 / 2}-25.406 \mathrm{mN}$, and the one from cubecorner $F_{\mathrm{N}-1}=25.124 h^{3 / 2}-1.0337$ and $F_{\mathrm{N}-2}=31.417 h^{3 / 2}-7.665 \mathrm{mN}$.

The indentation onto Germanium (n11 - n12) [27] gives the regression lines for Berkovich $F_{\mathrm{N}-1}=97.083 h^{3 / 2}-1.9363$ and $F_{\mathrm{N}-2}=128.58 h^{3 / 2}-5.8364 \mathrm{mN}$. The $k_{2}$-value with Berkovich could not be reasonably determined from the published loading curve.

The loading curve $(\mu \mathrm{N}, \mathrm{nm})$ for the bulk of the $\mathrm{Cu}_{60} \mathrm{Zr}_{30} \mathrm{Ti}_{10}$ alloy (n13) [28] gives the regression lines $F_{\mathrm{N}-1}=2.1803 h^{3 / 2}-301.72$ and $F_{\mathrm{N}-2}=2.6791 h^{3 / 2}-1289.7$. The transition onset is at $4016.830 \mathrm{mN}$, and the endothermic transition energy is $967.275 \mu \mathrm{Nnm} / \Delta h$. A further transition onset follows at about $9000 \mu \mathrm{N}$ load.

The loading curve of vitreloy $(\mathrm{mN}, \mu \mathrm{m})$ with the cubecorner (n14) [29] gives the regression lines $F_{\mathrm{N}-1}=29.652 h^{3 / 2}-11.605$ and $F_{\mathrm{N}-2}=36.139 h^{3 / 2}-37.756$.

Table 3 collects the results primarily from glassy ceramics $(n 1-n 6)$ for which both Berkovich and cubecorner indentations have been published. For comparison it also contains 2C22 steel alloy (n7 - n8) with an intermediate behavior, nickel (n9 - n10) as a crystalline metal, germanium (n11 - n12) with partly unsuitable data, and the metallic glasses (n13) and (n14) with "free volume" pores or shear bands formation even though these are only indented with one indented type, each. For glassy vitreloy (n14), here with the $\Delta h$ normalization, we point to the possibility of partial crystallization and shear bands, as formed on the surface. It is one of the entropic metallic glasses with extremely high values of phase transition onset force and phase-transition energy. The examples (n13) and (n14) are necessary for pointing out that not all super-cooled amorphous materials solidify without pores or partial crystallization that allow for some pile-up. Such complications must also be taken care of. Fortunately, these features can now be easily determined by checking whether or not such material exhibits pile-up upon indentation or not.

The algebraically calculated mechanical results from loading curves of primarily glassy ceramics in Table 3 (n1 - n6) confirm the unfitted calculations from the regression lines of $F_{\mathrm{N}}$ vs $h^{3 / 2}$ plots (1) for final applied forces from $2.5 \mathrm{mN}$ (fused quartz) up to $600 \mathrm{mN}$ (Zerodur ${ }^{\circledR}$ ) or $500 \mathrm{mN}$ (vitreloy-105). This rests on the now available volumes of Berkovich and cubecorner indenters as a function 
of the penetration depth. All phase-transitions in Table 3 are endothermic and pile-up is not possible in the isotropic materials $(n 1-n 6)$ : they have no cleavage planes or channels for materials' slide. The crystalline porous alloy (n13) is an evident exception in this respect, due to pile-up formation via the pores. The $2 \mathrm{C} 22$ steel has still the very close correspondence with the phase-transition onset of both indenters.

Importantly, the earlier claimed 1 to 3 ratio of displaced material between Berkovich and cube corner ([18] [21]) is clearly disproved. Despite the same displaced volume, the ratios of the physical hardness $k_{1 \text {-Berkovich }} / k_{1-\text { cubecorner }}$ vary for the different glassy ceramics between 3.07 and 4.99 in Table 3 (n1 - n6). For crystalline nickel it is 5.09. It is thus not constant and cannot be related to the $\tan \beta$ ratio of the indenters (3.0705), due to the different force directions and force powers. The phase-transition onset depths of the glassy ceramics vary from 0.090 or $0.189 \mu \mathrm{m}$ of fused quartz to 0.137 or $0.362 \mu \mathrm{m}$ of sodium aluminosilicate. But due to the displaced volume equality, the phase-transition onset forces are for these ceramics equal between Berkovich and cubecorner. The results with the glassy ceramics are particularly remarkable when the strong variations of the normalized phase-transition work from $0.23-96.5 \mathrm{mN} \mu \mathrm{m} / \Delta h$ are considered. The comparably very low value of fused quartz in connection with the low transition depth is a serious but mostly still not realized burden of its use as calibration standard for nanoindentations. Actually it should only be used at forces below $1 \mathrm{mN}$ for correct calibrations with Berkovich indenter and the ISO 14577 standard requires urgent revision. Conversely, Zerodur would be qualified as an indentation standard by its very high normalized transition work of 87 or 96.5 $\mathrm{mN} \mu \mathrm{m} / \Delta h$ for resisting high mechanical and apparently also thermal stress in Ceran ${ }^{\circledR}$ cooking plates. The super-cooled $2 \mathrm{C} 22$ steel is with its $14.4 \mathrm{mN} \mu \mathrm{m} / \Delta h$ far away from that. Further published experimental curves are as yet missing. It is an interesting consequence of the energy conservation law and certainly an important tool for the ceramics industry.

The very closely identical values for the Berkovich and cubecorner volumes in Table 2 indicate that both indenters behave in the same way with the isotropic glassy ceramics that does not allow for slide of material that would require corrections for Equations (10) and (11). Corrections for tip rounding of sharp indenters and the compression of diamond indenters are also not necessary. These are not part of the mathematical Equations (9), (10), and (11) and they are at least very similar for both indenters. Our results are very close to the mathematical precision, because of the excellent mechanical qualities of the ceramics in question (e.g. Zerodur ${ }^{\circledR}$ ). Any slight deviations are the result of low tip-angle precision and lack of the original data set for the data evaluation. We deal here with deep indentations and the tested materials are not super-hard. The sharp tip apex will stay sharp and its inevitable rounding is corrected together with the surface effects by $F_{a}$ in (2). However, when indenting super-hard materials the low elastic compliance of diamond would require correction and the then in- 
creasing tip bluntness would have to be controlled over and over again with tapping mode AFM (but please not with the iterative penetration onto fused quartz).

We must now discuss the reasons for the differences between Berkovich and cubecorner indentations. Unlike equal displaced volumes $V$, the areas $3 A_{\text {side }}$ of the Berkovich are about 1.3 times larger than the areas $3 A_{\text {side }}$ of the cubecorner at the same force (Table 2), despite the much deeper penetration of the latter. The created polymorph cover upon phase transition will therefore be broader at the cubecorner side-areas than at the Berkovich side-areas at the same force. The different side face areas (13) and (14) do not change the phase-transition onset forces but they are certainly part of the differences between the transition works, as performed by Berkovich or cubecorner indentation. But the normalized phase-transition energy (8) values do not precisely correspond after division by the respective pyramidal surfaces $3 A_{\text {side }}(13)$ and (14). The smaller transition energy value with the Berkovich indenter at the isotropic ceramics is thus not only due to its larger surface at the same indentation force. The cubecorner has to compress the same amount of the transformed polymorph from its smaller $3 A_{\text {side }}$-area (Table 2) forming a broader cover over a much longer distance. And the polymorph is mostly less compliant than the bulk. Thus, the cubecorner has to add more compression energy for the same amount of transformed material to the endothermic phase-transition energy than the Berkovich. But this sidearea influence cannot be the only reason for these differences of the $W_{\text {tansition }}$ values between the different indenters.

Perhaps more important than the side-area influence, is the force direction influence. The three-sided pyramids are three-sided "wedges" with the angle $\beta$ (Figure $3)$. The sideward force component $F_{\text {side }}$ with influence to the phase-transition onset calculates with the simple wedge-force formula $F_{\text {side }}=F_{\mathrm{N} \text {-onset }} / 2 \sin \beta$. For the phase-transition onset of e.g. Zerodur with Berkovich the direction of this force component is at the angle of $90-65.27=24.73^{\circ}$. One calculates $F_{\text {side-Berkovich }}=248.2246 / 2 \sin 65.27=136.6440 \mathrm{mN}$. For the cubecorner phasetransition onset the direction of that force component is $90-35.246=54.754^{\circ}$. One calculates $F_{\text {side-cubecorner }}=250.1074 / 2 \sin 35.246=217.2346 \mathrm{mN} \quad(1.59$ times stronger than Berkovich). That is in both cases for every one of the 3 side-faces. At the phase-transition onset the cubecorner is roughly twice as deep as the Berkovich and the cube corner adds more force, which increases the endothermic transition energy over the one from the Berkovich. That is indeed observed for the glassy ceramics. The force direction is by far steeper for Berkovich and it compresses further down with lower resulting force than the cubecorner that compresses in a flatter way with higher resulting force. The graphical analysis provides $13.7^{\circ}$ for Berkovich and $25.5^{\circ}$ for cubecorner for the direction of the resulting force with respect to the indenter central line from the force parallelogram.

Importantly, its shallower compression direction is now also the straightforward explanation for the more efficient cubecorner indentations for fracture 
toughness determinations via cracks, when compared to Berkovich. These observations and explanations clearly disprove the claim of [21] with compression of material at the apex of the cubecorner. Surprisingly, recent authors did not consider the force directional action when constructing their theories and simulations that are thus in error. Also the equal phase-transition onset forces for glassy ceramics and the different transition energies with the higher value from the cubecorner and the lower physical hardness $k(1),(2)$ of the cubecorner are so reliably explained and comprehended. It's the force and the force direction from indenters that counts.

There are no problems with slide compliance in glassy ceramics. We note that all of these glasses indent without pile-up. In the absence of pile-up the phasetransition force must be equal for both indenters, and the quality of such indentations can be checked with this fact.

The novel mathematical insights are used without any correction necessity for the unprecedented facts and applications for glassy ceramics in Table 3 (n1 n6). These basic insights are also valid for the further materials in Table 3 (n7 $\mathrm{n} 8$ ), but further influences require corrections that are not included in the basic mathematical treatment. Pile-up and internal slide along cleavage planes would require their being reported, volumetrically measured, and crystallographically analyzed. Nothing of that is known yet, so that we need more research for developing techniques for the necessary corrections. A first partial solution is presented in Section 3.5.2. The steel in Table $3(n 7-n 8)$ is a borderline case, where the phase-transition onset force is almost the same for Berkovich and cubecorner. But the phase-transition energy value is now larger for Berkovich than for cubecorner. The differences with the ceramic examples increase enormously with anisotropic crystalline nickel with slide effects in Table 3 (n9 - n10). It provides a twofold difference in the phase-transition onset force between the indenters. Furthermore, the phase-transition energy is more than 3 times larger for Berkovich than for cube corner in that case. For an explanation one should at first know the different pile-up volumes for both indenters at the same force. Further difficulties with pile-up are discussed in Section 3.5.1. There is an unfortunate lack of comparative loading curves in that respect.

Before the discussion of pile-up in Section 3.5 we have to take care of reliable experimental data that must not be simulating fitted ones. The mathematical calculation of indenter volumes reveals unexpected further common errors by treating the pyramidal indenters as pseudo-cones. Unfortunately, equal base-area cones are not equivalent to the pyramids.

\subsection{The Undue Treatment of Pyramids as Pseudo-Cones}

The now available volumes and side-areas of pyramidal indenters open new questions for the validity of the widespread use of pseudo-cones with equal basearea for the pyramids. ISO 14577 using [5], and so textbooks, and the so guided indentation research, including industrial technical applications, still believe in 
indentation ISO hardness $H$. Unfortunately, that relies on "projected area", or in refined form "contact area". The latter is obtained via two iterations with 3 and then with 8 free parameters. Furthermore, it became common practice to treat the indenting pyramids as pseudo-cones with equal basal-area. This allows for easier calculations and huge savings in computer time for iterating simulations (e.g. [22] and [30]). The (half) opening angles of the pseudo-cones had been so calculated that the projected indentation area is indeed the same as with the corresponding pyramids. These almost always used angles are $70.2996^{\circ}$ for Berkovich and $42.28^{\circ}$ for cubecorner. But do pseudo-cone pyramids really penetrate to the same depths as pyramids? The previous Section 3.3 gives the answer and that is no! These so-called "effective" or "equivalent" models for pyramids are in fact pyramidal phantoms. Their sideward force component direction angles are $90-70.2996=19.7004^{\circ}\left(\right.$ instead of $\left.24.73^{\circ}\right)$ and $90-42.28=47.72^{\circ}$ (instead of $54.736^{\circ}$ ) for the Berkovich phantom and for the cubecorner phantom, respectively. This leads to errors in ISO-hardness and ISO-elastic modulus. Both pyramidal phantoms would give considerably flatter force direction and force power than the respective pyramids at the same applied force $F_{\mathrm{N}}$. The resulting pseudo-cone phantom errors of the numerical data are huge. One may compare the depth differences at $600 \mathrm{mN}$ load onto Zerodur (Table 3) between Berkovich and cubecorner of $2.73 \mu \mathrm{m}$, which depends on about $30^{\circ}$ angle difference of the sideward force angle contribution. Here we have $5^{\circ}$ or $7^{\circ}$ smaller angles than the pyramids, which influences both depth and resulting force, as calculated via (15) and (16) at the same applied force $F_{\mathrm{N}}$. Clearly, these "equivalent cones" are not at all equivalent to the pyramids. Our evident volume, side-area, and directional force effects have never been considered. The false use of the pseudo-cone phantoms has therefore never been challenged before.

The mathematical volume and side-area of the pseudo-cone phantoms as a function of depth calculates again straightforwardly. We use the corresponding half-opening angles $\alpha=70.2996^{\circ}$ and $42.28^{\circ}$ for Berkovich and cubecorner phantomes, respectively. For the cone volume as a function of depth one starts with $V_{\text {cone }}=\pi r^{2} h_{\text {cone }} / 3$ and substitutes the basal $r$ by $h_{\text {cone }}$ via $\left.\tan \alpha=r / h_{\text {cone }}\right)$ to obtain without difficulty Equation (15). For the side-area of cones one uses $A_{\text {side }}=\pi r s$, where $s$ is the side length. With $\sin \alpha=h_{\text {cone }} / r$ and $\tan \alpha=r / h$ followed by substitutions of $r$ Equation (16) is obtained.

$$
\begin{gathered}
V_{\text {pseudocone }}=\pi \tan ^{2} \alpha h_{\text {cone }}^{3} / 3 \\
A_{\text {pseudocone }}=\pi \tan ^{2} \alpha h_{\text {cone }}^{2} / \sin \alpha
\end{gathered}
$$

Apparently nobody figured out yet that such "equivalent cones" have not the same volume at the same force and angle as the pyramids. We test here with the pseudo-cone phantom of $70.2996^{\circ}$ [31] for Berkovich. For cubecorner we test with the well-known old $42.28^{\circ}$ but not with new $40.018^{\circ}$, as claimed in [22] (for avoiding an error of $16.2 \%$ ). The numerical factors for $V_{\text {pseudocone }}$ in (15) for mimicking Berkovich are 8.168037 and for mimicking cubecorner (we test with the 
$42,28^{\circ}$ value) it is 0.865836 . These numerical volume factors are within at most $0.04 \%$ indistinguishable between pyramids and pseudo-cones. However, the respective volumes are different after their multiplication with the depth ${ }^{3}$ values that are smaller with the pseudo-cones due to the lower depths (cf Berkovich and cubecorner).

The numerical factors of $A_{\text {pseudocone }}$ (16) are 26.02765 for the Berkovich phantom and 3.86100 for the cubecorner phantom. The phantom values are thus smaller by $3.5 \%$ and by $14.2 \%$ respectively than those of the pyramids in Equation (13) and (14). This would after multiplication with the smaller depths ${ }^{2}$ obtain smaller expected areas than with the pyramidal indents. Therefore, the equalization of pseudo-cone with pyramid and all simulations on that basis are in severe error also for that reason. The simulations of [22] are cited above. These facts are still not recognized by the simulation groups from [21] [26] [30] [31], and many others.

The incorrect use of pseudo-cone pyramids add to the further flaws of published experimental indentations that are now easily recognized by novel straightforward testing. They appear often "influenced" by the hard to understand claims of the Oliver-Pharr group [18]. For example, further undue approaches argued with images of "broader" Berkovich coverage and "smaller" cubecorner coverage with complying material (actually after phase-transition onset it is polymorph). Their pressure distribution images [21] are more than questionable. Such claims are totally misleading and so are the extremely complicated discussions in that paper.

There were no reasons for challenging the pseudo cone phantom claims in 2017 [32] [33], and [34]. The unexpected new results exclude the use of the so-called "equivalent cone angles" from that time and we apologize for their then bona fide use. All applications and conclusions are still correct in these 3 publications. Only the numerical values that depend on the $\alpha_{\text {cone }}$ angles of the pseudo-cone pyramids cones require correction.

\subsection{The Pile-Up Influence}

Pile-up upon indentation onto anisotropic nickel could not be corrected in Section 3.3, Table 3 ( $\mathrm{n} 9$ - n10) due to pile-up formation. It is therefore now important to discuss the pile-up formations and effects. Anisotropic crystals with cleavage-planes and channels allow for sliding of materials along these and if they end at the indented surface, it is pile-up. Crystals are therefore the more compliant the better the force direction angle corresponds with the cleavage plane directions. That explains the anisotropy of indentation results upon indentations onto different crystallographic faces. Such sliding costs penetration depth that must be corrected for the penetration volume and it differs from material to material. Not all cleavage planes and channels end at the indented surface. There is also hidden sliding, and there are larger and smaller cleavage planes and channels with different sliding qualities in various directions. Sliding is also responsible for the differences between Berkovich and cubecorner inden- 
tations. This complicates the issue and requires detailed crystallography. The detailed crystallographic analysis of slide plane influences on not iterated physical hardness, phase-transition onset, and its energy has already been published for silicon onto (001) and (100), or $\alpha$-iron onto (100), (110), and (111), or Ca$\mathrm{CO}_{3}$ (three faces) in [16], or in the case of $\alpha$-quartz onto 4 different faces. The anisotropy is explained with differently orientated channels in [12]. Crystallographic analysis of their 3D interlocking in crystals is important. One must detect the face of the crystal opposite to the skew indenter face. There might be channels that are too small for sliding but these facilitate the compliance and also induce anisotropy [12]. Some of these faces and channels end at the indented surface, others at the side faces. It might be even possible to find indentation directions on surfaces without exit from these, and sliding might end within the crystal. Furthermore, micro-porous materials will also produce pile-up.

There is no need to consider sink-in [1]. The depth of indentations is always measured from the height of the initial flat surface. And sharp pyramids like Berkovich or cubecorner indenters penetrate immediately upon contact. Their tip rounding can be neglected at sufficiently deep penetrations. Also initial surface effects are cut off by taking care of the axis cut in $F_{\mathrm{N}}$ vs $h^{3 / 2}$ plots [2] (Section 2).

\subsubsection{The Common Interpretation of Pile-Up with Iterated Mechanical Properties}

It is clear that the pile-up and hidden slide volume has to be added to the inverted pyramid volume, so that the mathematical depth requires correction for it. But the common ISO 14577 and ASTM guided discussion of the pile-up phenomenon for anisotropic materials does not consider cleavage planes and channels. It claims friction at the indenter instead. This must again be challenged, as it is not intelligible. The published ISO 14577 and ASTM guided publications claim that "no significant difference occurs between cubecorner and Berkovich measurements". This has been "tested" for iterated ISO-modulus $E_{\mathrm{r}}$ and also for iterated ISO-hardness $H$ with the claim: "no fundamental difference is observed" [35]. Unfortunately, no experimental loading curves were supplied for obtaining not iterated data. The published data for 8 very different materials are within experimental error, on the basis of the $3+8$ free parameters in the two consecutive iterations. This common ISO-ASTM procedure is still commonly used. Unfortunately, these claims would seem to include that numerous other mechanical qualities might also be without "significant difference". Also phrases like "Friction does not significantly influence the simulation of the load-displacement response in indentations" [36] do not deny the challenged friction approach. And phrases like "Pile-up occurs due to isovolumetric plastic flow parallel to the indenter surface when the strain is concentrated directly below the indenter" [37] do not explain anything. And so are the strange theories that are connected with it.

The averaged pile-up height around the indentation in [38] is obtained by AFM measurements of the indented surface, but this does not include the vo- 
lume of the pile-up. Pile-up falsifies the ISO hardness $H$ and ISO modulus $E_{\mathrm{r}}$ enormously ( $H$ up to $60 \%$ error, sometimes even $80 \%$ error, and $E_{\mathrm{r}}$ up to $30 \%$ error), because the "contact depth is falsified". It is well-known, but most colleagues live with it. For example [39] complains it. But ISO- $H$ and ISO- $E_{\mathrm{r}}$ values are nevertheless used in its finite element simulations. The current argumentation includes $h_{\mathrm{f}} / h_{\max }$ ratio ( $h_{\mathrm{f}}$ for $h_{\max }+h_{\text {pile-up }}$ ), strain hardening coefficient $\mathrm{n}$ and $E / \sigma_{y}$, averaged pile-up heights (instead of pile-up volumes), "indentation size effects" (instead of phase-transitions), and work hardening. All of that is published instead of considering the papers [1] and [2]. The contents of these were also long before discussed in numerous worldwide conferences.

Unfortunately, the faulty simulations of [39] stimulated the belief in even more extended simulations and iterations with finite element analysis. These include in their calculation not less than the complexity of $h_{\mathrm{f}} / h_{\max }$ ratios, ISOhardness $H$, ISO-modulus $E_{\mathrm{r}}$, contact stiffness $S$, indentation size effect (ISE), pile-up height, strain hardening exponent, strain hardening coefficient, stress and strain, yield point, yield strain, yield stress, Poisson's ratio, equivalent strain energy elastic and elastoplastic, tip angle, cone model of Berkovich, deformed volume with Johnson's cavity model, total indentation work, and friction coefficient with numerous fitting constants [37]. Numerous of these building blocs are iterated ones. These more than complicated techniques are included in "inverse estimation iteration techniques".

Enormous iterations are used for describing the pile-up topography with an algorithm, but still not for the pile-up volume. Furthermore, there are different contradicting approaches and simulations. None of them help for understanding the pile-up events. And none of them invoke cleavage planes and channels of the materials. Such simulations did not distinguish between isotropic and anisotropic solids. Thus, all of these enormous diverting simulations and iterations are obsolete.

\subsubsection{The Solution with Pile-Up Volume along Cleavage Planes}

Fortunately, the physical hardness $k\left[F_{\mathrm{N}} / h^{3 / 2}\right]$ of pyramidal and conical (with cones) indentation, which is in accordance with the energy conservation rule [1], [2], is not invalidated by any pile-up. The latter is however influencing the steepness of the straight regression line. The failure of the simulations as challenged in Section 3.5.1 derives from their inability to take care of cleavage planes and channels, of directional force effects, and of phase-transitions. All pile-up considerations must additionally care for "free volume" pores, which also enable slide possibilities upon stress.

We must now more detailed discuss the reasons for pile-up. Cleavage-planes and or channels for the sliding of locally stressed materials are always present in the anisotropic crystalline matter. Slide possibilities along cleavage planes and channels must always be considered. They are revealed from proper crystallographic analysis and can facilitate the crystals compliance. Ample examples with crystal packing images are available in [12] [16], and [40]. 
In order to prove the slide of material along the cleavage planes for pile-up, we looked for a suitable system with only one type of parallel cleavage planes that can be checked from different directions. And we determined the volume and shape of the pile-up. The organic molecule thiohydantoin crystallizes with parallel not interlocked molecular sheets in space group $\left(P 2_{1} / c\right)$ and melts at $230^{\circ} \mathrm{C}$. It is a particularly suitable example with $66^{\circ}$ steep unimolecular sheets that have the single cleavage plane direction for slide between them. The sheets and thus the parallel cleavage planes end on the (110) surface.

The model in Figure 4 shows the different indentations with a cube corner indenter upon ramp scratching (indentation with increasing force along a predefined horizontal distance) in direction (a), (b), (c), and (d) from $0-400 \mu \mathrm{N}$ load over a distance of $10 \mu \mathrm{m}$. The pile-up can now be freely selected from none, equal, and equal with perhaps slightly more than equal, as compared with the depression volume. The last possibility would mean that some broken edges added to the pile-up. The impressive interactive color images are freely available in [41].

The scratch against the sloping (dir. c) (Figure 4) provides almost no pile-up, because the sheets are pushed down. The scratch with the sloping (dir. a) provides pile-up to both sides with a pile-up volume equal or perhaps slightly larger than the depression volume. Due to overlapping pile-up it does not leave well defined reference edges and corners. The volume can thus not precisely be quantified with AFM and the bearing analysis routine [8] [9] in that case. Most interesting is the scratch along the sheets' direction. It depends on the orientation of the crystal whether all of the pile-up occurs to the left (dir. b) or upon turn of the crystal by $180^{\circ}$ to the right side (dir. d) of the scratch direction onto the (110) face. In these cases the pile-up volumes are equal to the depression volume. For example, the ratio for the measured dir. $\mathrm{b}$ direction is $2.661 \mu \mathrm{m}^{3}$ (pile-up) to $2.588 \mu \mathrm{m}^{3}$ (depression). No pileup is formed by normal indentation onto the (10-2) faces left or right of the crystal (Figure 4). The sliding migrations from the penetrated sheets cannot exit the crystal in these cases. Such internal slide is "lost pile-up" that also detracts from the mathematical depth and volume values.
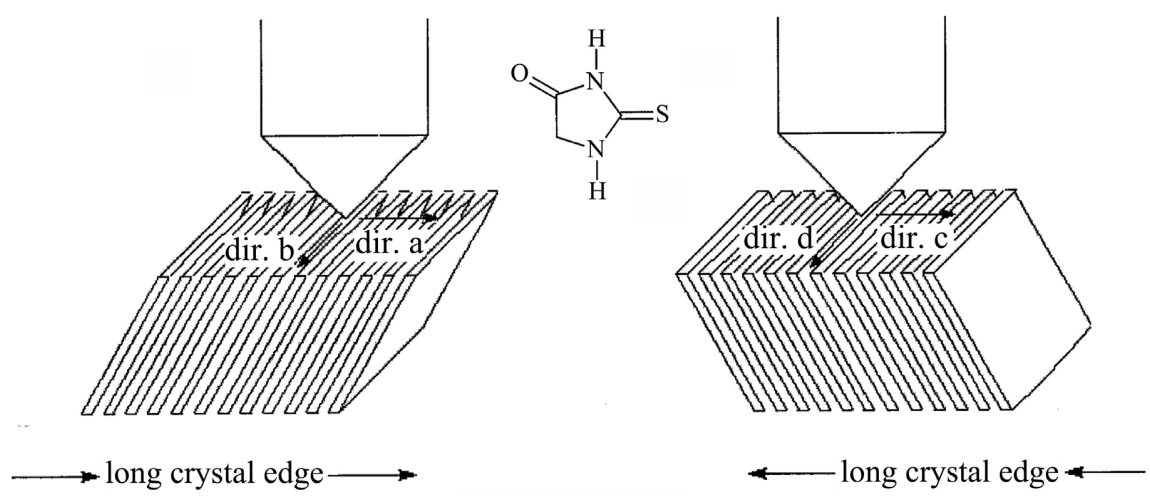

Figure 4. Two geometric models at $0^{\circ}$ and $180^{\circ}$ orientation of the $66^{\circ}$ skew monolayer sheet packing of thiohydantoin, indicating the four different indentation orientations for the ramp scratches with cubecorner indentation; the model is redrawn in part from [41]. 
This model is fundamental and convincing due to only one skew cleavage plane direction between unimolecular sheets. Further examples for pile-up to the right or to the left hand side, just by turning the crystals by $180^{\circ}$, are known from [41] and [42] for anthracene or for tetraphenylethene with their skew cleavage plane directions. These examples rest on their proper crystal structure analyses.

The situation is more complicated for crystals with more than one type of cleavage directions. All of them require identification and indentation in the respective directions. Such as yet missing studies with crystalline materials will open the possibility to develop the corrections of the undeniable mathematical formulas for indenter volume and side-face. Practical applications will become unavoidable with that endeavor.

It is essential to always calculate the pile-up volumes (not only the average height) with the bearing analysis routine [8] [9] on the basis of the plane through the respective corners. Unfortunately, such volume calculations are as yet not performed at the expense of only image topologies with AFM or of their simulation by using finite element calculation routines. Our striking results with thiohydantoin, anthracene, and tetraphenylethene disprove the validity of conclusions from pile-up height simulations. It's the volume that counts!

In fact most crystalline materials of all types are much more complicated than thiohydantoin, anthracene, and tetraphenylethene with numerous cleavage planes in different directions for sliding materials. All of these must be studied by indentations onto various faces to experimentally check their efficiency (angle and width). It might also be possible to find directions without pile-up. Hidden sliding occurs when cleavage planes or sufficiently wide channels cross the indentation direction.

A special case is remote pile-up about 50 to $200 \mu \mathrm{m}$ from the indentation center. The Vickers impression onto crown glass at $50 \mathrm{~N}$ load is an example where these are symmetrically in line at right angle [23]. Clearly, there is materials slide along submicron cracks, as formed upon stress relief. In the case of flint glass such submicron cracks are unsymmetrical and so are the long-range pile-up features. Both glasses are amorphous and brittle though.

We remind here another long-range crack initiation and completion with the detection of the two-step nucleation in $\mathrm{NaCl}$ that happened upon depth-sensing Vickers indentation with the polymorph after its 5 th phase-transition at $24.43 \mathrm{~N}$ load [17]. At $28.5 \mathrm{~N}$ load and $6.1 \mathrm{~mm}$ (!) from the indentation center occurred a mini-thin crack trace. It was detected at 5000X magnification with the $3 \mathrm{D}$ digital microscope. This tiny micro-crack nucleated a $5 \mu \mathrm{m}$ short pre-crack with 1.68 $\mu \mathrm{m}$ width. Upon the further load the crack resumed from the continued micro-crack and completed without pile-up, while the load was continuously increased. At $29.8 \mathrm{~N}$ load the macroscopic crack was $8.5 \mu \mathrm{m}$ wide. At $50 \mathrm{~N}$ load after $5.8 \mathrm{~mm}$ length the crack was $11.2 \mu \mathrm{m}$ wide and $3.12 \mu \mathrm{m}$ deep at the exit from the sample end. Clearly, long-range release of the stress due to the compacting 
imposed by the indenter can be released along micro-cracks. Depending on the material there can be crack nucleation (crystal) or distant pile-up (glass).

Long-range pile-up is also possible with porous materials (e.g. the metallic glass $\mathrm{Cu}_{60} \mathrm{Zr}_{30} \mathrm{Ti}_{10}$ with pores in Table 3 (n13). Isotropic materials like Zerodur or fused quartz help themselves by phase transition with polymorphs around the indenter.

The compression of the diamond indenter that is not corrected for, does not visibly affect the use of (9), as shown by the results of Table 3. Also the amorphous to amorphous phase-transitions (from floppy to rigid [4]) do not affect the results. But in case of pile-up we do not know yet. Again, phase transitions are not seen in $F_{\mathrm{N}}$ vs $h$ loading curves but only in there-from calculated $F_{\mathrm{N}}$ vs $h^{3 / 2}$ plots.

All of the here presented unprecedented facts can not be recognized by fitting simulations. Unfortunately they are thus unthinkable by the common ISO 14577ASTM standards that are still confined to the Oliver-Pharr iterations of 1992. And these are not obeying the energy conservation law (see [1] [2]).

Further comparisons of Berkovich and cubecorner indentations onto crystalline materials with forces well beyond the phase-transition onset are badly required.

\section{Conclusions}

The consideration of volume, side-area, and resulting force direction of pyramidal or conical indenters straightforwardly replaces numerous incorrect common dogmas with easy self-evident unprecedented cognition. This paper describes novelties from the mathematical treatment of indentations in great detail, so that it can be easily reproduced. Poor experimental data that do not give straight line $F_{\mathrm{N}}$ vs $h^{3 / 2}$ plots or fitted false linear $F_{\mathrm{N}}$ vs $h^{2}$ ones can be and must be excluded from consideration. Also, instrumental miscalibration, or denial of phase-transitions from [5] is still falsifying all of the 6 common calibration standards as reveled in [12]. Incredibly, the long revealed phase transitions from all of them are still disregarded in the ISO 14577-ASTM standards! ISO- $H$ and ISO- $E_{\mathrm{r}}$ are unphysical and thus very dangerous characterizations of technical materials. Only undeniable calculation rules characterize phase transition onsets (depth and force) and transition energies. The advanced normalization of phasetransition energies per $\Delta h=\left(h_{\text {polymorph }}-h_{\text {onset }}\right)$ allows for mathematical comparison of Berkovich and cubecorner energies. The reliability of the precise calculations has been shown. They do not increase experimental errors by rounding errors.

Isotropic ceramics proceed without complications. They are particularly useful for understanding the differences between pyramidal or conical indentations. The formulas for the volumes, side-areas for pyramids and for cones as functions of penetration depth, and the angle dependent force directions are straightforwardly deduced and used. The sidewise wedge-type force of the indented cubecorner is 1.59 times stronger than the one of the Berkovich indenter at the same 
applied force. Thus, cubecorner with higher force, flatter resulting force direction, and lower side-area is better for fracture toughness determinations than Berkovich at the same applied force.

The undeniable mathematical formulas are experimentally realized for ceramics lacking pile-up. The previous widely acknowledged claims of 3-times larger displaced volume for cubecorner as compared to Berkovich at the same indentation force is mathematically and physically disproved without using any iteration.

The very widespread mimicking of pyramids with "equivalent" cone angles for the same projected indentation area is in severe error (depth, force, angle, hardness, etc) and must be urgently cancelled. The straightforward consideration of basic physics and mathematics identifies the chimera.

The occurrence of pile-up with anisotropic crystalline material creates indentation volumes with different amounts for Berkovich and cubecorner. The pileup requires a completely new understanding. The previous view of pileup claiming friction of indenter with material and the unintelligible nebulous claims of materials "slip" are straightforwardly rejected. Both pile-up to the surface and hidden "pile-up" within the materials use cleavage planes or channels or cracks for sliding under the mechanical stress. Several of these paths in different directions and with different widths produce different results on different faces (anisotropy) and with different indenters (force direction). That is demonstrated using the new insights from this paper and by stressing the crystallographic techniques. Pile-up is definitely not produced by friction with the indenter. The non-appreciation of the crystallographic facts by the ISO 14577-ASTM indenter community led to extremely complicated iterative worthless simulations that are not helpful for materials mechanics. Iterative pile-up simulations are extremely dangerous when applied to technical materials. These facts are experimentally confirmed with indentations including pile-up volume measurement. Pile-up management has been exemplified using skew single cleavage plane orientations in crystals.

The new insights from the indenter volume and side-area formulas have numerous practical applications. The arithmetic equations are valid both for isotropic and for anisotropic materials, covering physical hardness, initial surface effects, and phase-transition-onsets, -forces, and -energies. The comparison of Berkovich and cubecorner indenters at the same force has the physical indentation hardness $\left(\mathrm{mN} / \mu \mathrm{m}^{3 / 2}\right)$ always smaller with the cubecorner, due to the deeper penetration. Only the ratio variations cannot be judged without further experimental data.

For isotropic materials the necessary phase-transition onset forces are equal for Berkovich and cubecorner, but the phase-transition energies are larger for the cubecorner due to its smaller $\beta$-angle (center line to side face) with flatter direction of the higher resulting force from the smaller side-area. For anisotropic materials the comparison of the different indenters is less predictable, because 
pile-up and internal sliding volume has to be added to the impression volume, and the applied force to depth ratio is decreased. Different resulting forces meet the existing cleavage planes and channels at a different angle. Much more experimental research is required for finding appropriate predictive rules. The necessity to measure any pile-up volume (not only AFM topography) and to reveal cleavage planes, channels, and pores is stressed.

The previous errors and the ones from non-consideration of phase-transitions must and can be corrected with the novel insights from this publication. But there is presently a lack of comparative indentions with different indenters onto crystalline materials with applied forces beyond their phase-transition onsets. Further research on these lines will help for a safer daily life.

\section{Outlook}

The present author asked ISO officials for early revision of ISO 14577, but that takes time for various non-mathematical reasons.

The unprecedented use of volume, side area, and force direction of pyramidal and conical indentations open numerous unexpected fields of research and unexplored applications. The background is undeniable calculation rules, but never simulative fitting of experimental data on the basis of incredibly difficult "theories", which still violate the energy conservation law from the beginning.

The here described first novel results require further development with new experiments and development of computer programs beyond the common Ex$\mathrm{cel}^{\circledR}$ features. Fast data calculation in huge comparable indentation series will enable the development of technical materials with much better and physically correct mechanical properties. The data validity checks remain with the $F_{\mathrm{N}}$ vs $h^{3 / 2}$ plot. Examples are improved light alloys with systematically changing compositions for much better mechanical properties, particularly in view of phasetransition unsteadiness under load.

The indentation science and the involved industry must try to discard iterative simulations and try to correct the characterization of technical materials. It must no longer trust in unphysical though still enforcing ISO 14577-ASTM hardness and modulus with their numerous there from deduced and perpetuated unphysical materials parameters. We remind here the broken propellers-blades in front of the turbines hitting the fuselage of airliners, and also the hundreds of grounded airliners since 2019 with cracks at the fuselage, including cracks even at the pickle forks between wings and fuselage, most likely due to constructions with TiAlX alloy. And there were still several catastrophic airliner accidents. The TiAlX alloys exhibit comparably low phase-transition onset forces and energies, forming polymorph interfaces as sites for crack nucleation upon mechanical stress. Considerably improved alloys have to be immediately developed on the physical and mathematical basis, not only for the aviation industry.

Furthermore, unsolved problems promise unexpected new advancements and applications. The mathematical comparison of physical hardness $\left(k\right.$-value $\left.F_{\mathbb{N}} / h^{3 / 2}\right)$ 
between different indenters requires further investigations with new comparative indentation experiments. The angle $\beta$ (Figure 3) must certainly play an important role in that endeavor. Correction of the indentation volume with the pile-up volumes on all different materials surfaces can be helpful for structural decisions. Such studies promise important new materials properties that are not at all available by iterations.

It is to be hoped that young researchers, who are not caught with extremely complicated simulations and iterative data fittings, will continue with using undeniable self-explaining mathematics for the indentation research. By doing so, they will reveal further physical effects that are responsible for the transition energy differences between Berkovich and cubecorner. Further pyramidal or conical indenters for anisotropic materials should be included.

\section{Conflicts of Interest}

The author declares no conflicts of interest regarding the publication of this paper.

\section{References}

[1] Kaupp, G. (2013) Penetration Resistance: A New Approach to the Energetics of Indentations. Scanning, 35, 392-401. https://doi.org/10.1002/sca.21080

[2] Kaupp, G. (2016) The Physical Foundation of $F_{\mathrm{N}}=k h^{3 / 2}$ for Conical/Pyramidal Indentation Loading Curves. Scanning, 38, 177-179. https://doi.org/10.1002/sca.21223

[3] Kaupp, G. and Naimi-Jamal, M.R. (2004) Nanoscratching on Surfaces: the Relationships between Lateral Force, Normal Force and Normal Displacement. International Journal of Materials Research, 95, 297-305.

[4] Kaupp, G. and Naimi-Jamal, M.R. (2010) The Exponent 3/2 at Pyramidal Nanoindentations. Scanning, 32, 265-281. https://doi.org/10.1002/sca.20206

[5] Oliver, W.C. and Pharr, G.M. (1992) An Improved Technique for Determining Hardness and Elastic Modulus Using Load and Displacement Sensing Indentation Experiments. Journal of Materials Research, 7, 1564-1583.

https://doi.org/101557/JMR.1992.1564

[6] Kaupp, G. (2020) Indentation onto Stishovite $\left(\mathrm{SiO}_{2}\right), \mathrm{MgO}$, and a Covered Superalloy: "Pop-In" Repair, Phase-Transition Onsets, Polymorph Energies, and Transition-Energies. Advances in Materials Physics and Chemistry, 10, 77-95. https://doi.org/10.4236/ampc.2020.103007

[7] Kaupp, G. (2021) Undue Hardness/Modulus Ratio Claims Instead of Physical Penetration Resistance and Applications with Mollusk Shells. Advances in Materials Physics and Chemistry, 11, 45-57. https://doi.org/10.4236/ampc.2021.112005

[8] Kaneko, R. and Hamada, E. (1993) Microwear Processes of Polymer Surfaces. Wear, 162-164, 370-377. https://doi.org/10.1016/0043-1648(93)90520

[9] Han, Y., Schmitt, S. and Friedrich, K. (1999) Nanoscale Indentation and Scratch of Short Carbon Fiber Reinforced PEEK/PTFE Composite Blend by Atomic Force Microscope Lithography. Applied Composite Materials, 6, 1-18. https://doi.org/10.1023/A:1008812915701

[10] Oila, A. and Bull, S.J. (2003) Nanotesting in Gear Steels. Zeitschrift für Metallkunde, 94, 793-797. https://doi.org/10.3139/146.030793 
[11] Wang, X., Wang, J., He, Y., Wang, C., Zhong, L. and Mao, S.X. (2020) Unstable Twin in Body-Centered Cubic Tungsten Nanocrystals. Nature Communications, 11, Article No. 2497. https://doi.org/10.1038/s41467-020-16349-8

[12] Kaupp, G. (2019) Phase-Transition Energies, New Characterization of Solid Materials and Anisotropy. Advances in Materials Physics and Chemistry, 9, 57-70. https://doi.org/10.4236/ampc.2019.94006

[13] Kaupp, G. (2014) Activation Energy of the Low-Load NaCl Transition from Nanoindentation Loading Curves. Scanning, 36, 582-589. https://doi.org/10.1002/sca.21158

[14] Kaupp, G. (2019) The Loading Curve of Spherical Indentations Is Not a Parabola and Flat Punch Is Linear. Advances in Materials Physics and Chemistry, 9, 141-157. https://doi.org/10.4236/ampc.2019.99012

[15] Kaupp, G. (2020) Real and Fitted Spherical Indentations. Advances in Materials Physics and Chemistry, 10, 207-229. https://doi.org/10.4236/ampc.2020.1010016

[16] Kaupp, G. (2019) Physical Nanoindentation: From Penetration Resistance to PhaseTransition Energies. Advances in Materials Physics and Chemistry, 9, 103-122. https://doi.org/10.4236/ampc.2019.96009

[17] Kaupp, G. (2018) Six Polymorphs of Sodium Chloride upon Depth-Sensing Macroindentation with Unusual Long-Range Cracks Requiring $30 \mathrm{~N}$ Load. Journal of Material Sciences and Engineering, 7, 473-483. https://doi.org/10.4172/2169-0022.1000473

[18] Harding, D.S., Oliver, W.C. and Pharr, G.M. (1994) Cracking during Nanoindentation and Its Use in the Measurement of Fracture Toughness. MRS Online Proceedings Library, 356, 663-668. https://doi.org/10.1557/PROC-356-663

[19] Li, X., Jiang, L., Mohagheghian, I., Dear, J.P., Li, L. and Yan, Y. (2018) New Insights into Nanoindentation Crack Initiation in Ion-Exchanged Sodium Aluminosilicate Glass. Journal of the American Ceramic Society, 101, 2930-2940. . https://doi.org/10.1111/jacc.15465

[20] Wang, Y. (2016) Effects of Indenter Angle and Friction on the Mechanical Properties of Film Materials. Results in Physics, 6, 509-514. https://doi.org/10.1016/j.rinp.2016.08.008

[21] Bor, B., Giuntini, D., Domenech, B. Swain, M. and Schneider, G. (2019) Nanoindentation-Based Study of the Mechanical Behavior of Bulk Supercrystalline Ceramic-Organic Nanocomposites. Journal of the European Ceramic Society, 39, 3247-3256. https://doi.org/10.1016/j.jeurceramsoc.2019.03.053

[22] Scordaris, G., Bouzakis, K. and Charalampous, C. (2018) A Critical Review of FEM Models to Simulate the Nano-Impact Test on PVD Coatings. MATEC Web of Conferences, 188, Article ID: 04017. https://doi.org/10.1051/matecconf/201818804017

[23] Hervas, I., Montagne, A., van Gorp, A., Bentoumi, M., Thuault, A. and Iost, A. (2016) Fracture Toughness of Glasses and Hydroxyapatite: A Comparative Study of $7 \mathrm{Me}-$ thods by Using Vickers Indenter. Ceramics International, 42, 12740-12750. https://doi.org/10.1016/j.cermint.2016.05.030

[24] Naimi-Jamal, M.R. and Kaupp, G. (2005) Quantitative Evaluation of Nanoindents: Do We Need More Reliable Mechanical Parameters for the Characterization of Materials? International Journal of Materials Research, 96, 1226-1236. https://doi.org/10.3139/146.101166

[25] Breuils, J., Krier, J., Pelletier, H. and Mille, P. (2011) Identification, Using Nanoindentation Tests, of Mechanical Behaviour of a 2C22 Steel Presenting a Residual 
Stress State. Procedia Engineering 10, 3528-3533. https://doi.org/10.1016/j.proeng.2011.04.580

[26] Zhao, Y., Seok, M.Y., Lee, D.Y., Lee, J.A., Suh, J.Y. and Jang, J.I. (2016) Hydrogen-Induced Softening in Nanocrystalline Ni Investigated by Nanoindentation. Philosophical Magazine, 96, 3442-3450. https://doi.org/10.1080/14786435.2016.1159743

[27] Jang, J. (2005) Evidence for Nanoindentation-Induced Phase Transformations in Germanium. Applied Physics Letters, 86, Article ID: 131907. https://doi.org/10.1063/1.1894588

[28] Jiang, W.H., Liu, F.X., Wang, Y.D., Zhang, H.F., Choo, H. and Liaw, P.K. (2006) Comparison of Mechanical Behavior between Bulk and Ribbon Cu-Based Metallic Glasses. Materials Science and Engineering: A, 430, 350-354. https://doi.org/10.1016/j.msea.2006.05.042

[29] Moser, B., Löffler, J.F. and Michler, J. (2006) Discrete Deformation in Amorphous Metals: An in Situ SEM Indentation Study. Philosophical Magazine 86, 5715-5728. https://doi.org/10.1080/14786430600627301

[30] Huang, S. and Zhou, C. (2017) Modeling and Simulation of Nanoindentation. JOM, 69, 2256-2263. https://doi.org/10.1007/s11837-017-2541-1

[31] Bucaille, J.L., Stauss, S., Felder, E. and Michler, J. (2003) Determination of Plastic Properties of Metals by Instrumented Indentation Using Different Sharp Indenters. Acta Materialia, 51, 1663-1678. https://doi.org/10.1016/S1359-6454(02)00568-2

[32] Kaupp, G. (2017) The ISO Standard 14577 for Mechanics Violates the First Energy Law and Denies Physical Dimensions. Journal of Material Sciences and Engineering, 6, 321-328.

[33] Kaupp, G. (2017) Dilemma between Physics and ISO Elastic Indentation Modulus. Journal of Material Sciences and Engineering, 6, 402-405. . https://doi.10.4172/2169-0022.1000402

[34] Kaupp, G. (2017) Challenge of Industrial High-Load One-Point Hardness and of Depth Sensing Modulus. Journal of Material Sciences and Engineering, 6, 348-355. . https://doi.10.4172/2169-0022.1000348

[35] Chudoba, T., Schwaller, P., Rabe, R., Breguet, J.M. and Michler, J. (2006) Comparison of Nanoindentation Results Obtained with Berkovich and Cube-Corner Indenters. Philosophical Magazine, 86, 5265-5283. https://doi.org/10.1080/14786430600746424

[36] Bhattacharya, A.K. and Nix, W.D. (1988) Finite-Element Simulation of Indentation Experiments. International Journal of Solids and Structures, 24, 881-891. https://doi.org/10.1016/0020-7683(88)90039-X

[37] Goto, K., Watanabe, I. and Ohmura, T. (2020) Inverse Estimation Approach for Elastoplastic Properties Using the Load-Displacement Curve and Pile-Up Topography of a Single Berkovich Indentation. Materials and Design, 194, Article ID: 108925. https://doi.org/10.1016/j.matdes.2020.108925

[38] Zhu, L., Yu, B., Wang, H., Wang, C. and Yang, D. (2011) Measurement of Mechanical Properties of 1045 Steel with Significant Pile-Up by Sharp Indentation. Journal of Materials Science, 46, 1083-1086. https://doi.org/10.1007/s10853-010-4876-7

[39] Gale, J.D. and Achuthan, A. (2014) The Effect of Work-Hardening and Pile-Up on Nanoindentation Measurements. Journal of Materials Science, 49, 5066-5075. https://doi.org/10.1007/s10853-014-8213-4

[40] Kaupp, G. and Naimi-Jamal, M.R. (2013) Penetration Resistance and Penetrability 
in Pyramidal (Nano)Indentations. Scanning, 35, 88-111.

https://doi.org/10.1002/sca.21038

[41] Kaupp, G, Schmeyers, J. and Hangen, U.D. (2002) Anisotropic Molecular Movements in Organic Crystals by Mechanical Stress. Journal of Physical Organic Chemistry, 15, 307-313. https://doi.org/10.1002/poc.487

[42] Kaupp, G. and Naimi-Jamal, M.R. (2005) Mechanically Induced Molecular Migrations in Molecular Crystals. CrystEngComm, 7, 402-410.

https://doi.org/10.1039/b503208n 